\title{
POSITIVE MARGINS AND PRIMARY DECOMPOSITION
}

\author{
THOMAS KAHLE, JOHANNES RAUH AND SETH SULLIVANT
}

\begin{abstract}
We study random walks on contingency tables with fixed marginals, corresponding to a (log-linear) hierarchical model. If the set of allowed moves is not a Markov basis, then tables exist with the same marginals that are not connected. We study linear conditions on the values of the marginals that ensure that all tables in a given fiber are connected. We show that many graphical models have the positive margins property, which says that all fibers with strictly positive marginals are connected by the quadratic moves that correspond to conditional independence statements. The property persists under natural operations such as gluing along cliques, but we also construct examples of graphical models not enjoying this property. We also provide a negative answer to a question of Engström, Kahle and Sullivant by demonstrating that the global Markov ideal of the complete bipartite graph $K_{3,3}$ is not radical.

Our analysis of the positive margins property depends on computing the primary decomposition of the associated conditional independence ideal. The main technical results of the paper are primary decompositions of the conditional independence ideals of graphical models of the $N$-cycle and the complete bipartite graph $K_{2, N-2}$, with various restrictions on the size of the nodes.
\end{abstract}

1. Introduction. Let $\mathcal{B}$ be a finite subset of $\mathbf{Z}^{n}$, and consider the graph with vertex set $\mathbf{N}^{n}$ (here, $\mathbf{N}$ denotes the natural numbers including zero) and edges $(u, v)$ whenever $u-v \in \pm \mathcal{B}$. We want to study the connected components of this graph. Our motivation comes

2010 AMS Mathematics subject classification. Primary 13P10, 52B20, Secondary 05C81, 11P21, 60J22, 62J12.

Keywords and phrases. Algebraic statistics, Markov basis, connectivity of fibers, binomial primary decomposition.

The first author is supported by an EPDI fellowship. The second author is supported by the Volkswagen Foundation. The third author is supported by the David and Lucille Packard Foundation and the U.S. National Science Foundation (DMS 0954865).

Received by the editors on February 29, 2012, and in revised form on September 20, 2012. 
from Markov chain random walks on $\mathbf{N}^{n}$ using the elements in $\mathcal{B}$ as moves. If every edge in this graph has positive probability, then the connected components are the irreducible components of the Markov chain.

A necessary condition for $u, v \in \mathbf{N}^{n}$ to be connected by $\mathcal{B}$ is that their difference vector $u-v$ lies in the lattice $\mathbf{Z B}$ generated by $\mathcal{B}$. We want to know when this condition is sufficient. In this paper we assume that the lattice $\mathbf{Z B}$ is saturated, that is, it can be written as the integer kernel $\operatorname{ker}_{\mathbf{Z}} A$ of an integer matrix $A$. We do not require $\mathcal{B}$ to be a basis of $\mathbf{Z B}$-there can be more than $\operatorname{rank}(\mathbf{Z B})$ generators. For any $u \in \mathbf{N}^{n}$ we call $(u+\mathbf{Z B}) \cap \mathbf{N}^{n}$ the fiber of $u$. For example, in the statistical analysis of contingency tables, people are interested in the set of all contingency tables with given marginals. In this case, the matrix $A$ corresponds to the linear map that computes the marginals from a contingency table. Monte Carlo sampling techniques are then applied to compute approximate p-values in Fisher's exact test for conditional inference $[\mathbf{1 1}, \mathbf{1 2}]$.

In the literature, often the following inverse problem is studied: Given a saturated lattice and a point $u \in \mathbf{N}^{n}$, find a set $\mathcal{B}$ such that the fiber of $u$ is connected. Such a set is called a Markov subbasis in [6]. Ideally, one wants to compute a Markov basis, a finite set that connects all fibers at once.

The fundamental theorem of Markov bases (see [12, Theorem 1.3.6] and Theorem 2.2 below) implies that Markov bases can be found using computer algebra. Despite fast computers, excellent algorithms [23] and efficient implementations [1], computing Markov bases remains hard and is out of reach for many practical applications. Furthermore, since Markov bases are guaranteed to connect every fiber, they might be much larger than needed to connect a particular fixed given fiber. In this paper we study conditions on the fiber that certify that a given set of moves connects this fiber. In particular, we say that $\mathcal{B} \subseteq \operatorname{ker}_{\mathbf{Z}} A$ has the positive margins property with respect to the matrix $A$ if $(A u)_{i}>0$ for all $i$ implies that the fiber of $u$ is connected by $\mathcal{B}$. This property depends not only on the lattice $\operatorname{ker}_{\mathbf{Z}} A$ but also the particular matrix $A$.

The main focus in this paper is on lattices and moves associated to graphical models. For graphical models, there is a canonical set of "simple" moves, which correspond to the global Markov conditional 
independence statements. It has been observed that, for some models, if a contingency table $u$ has strictly positive margins, then these simple moves connect the fiber of $u[7]$. Similarly, for the no-three-way interaction model Bunea and Besag proved a positive margins property for a set of "simple" moves [5]. In the present paper, we perform a systematic study of the positive margins property for graphical models.

Connectivity of lattice walks can be studied with tools from commutative algebra using the following idea: Consider the binomial ideal

$$
I_{\mathcal{B}}=\left\langle p^{u_{+}}-p^{u_{-}}: u \in \mathcal{B}\right\rangle \subseteq \mathbf{k}\left[p_{1}, \ldots, p_{n}\right]
$$

where $\mathbf{k}$ is a field, $u=u^{+}-u^{-}$is the minimal support decomposition of $u$ into positive and negative parts, and $p^{v}=p_{1}^{v_{1}} \cdots p_{n}^{v_{n}}$. The following result is well known (see [10] and the references therein).

Proposition 1.1. Two points $u, v \in \mathbf{N}^{n}$ are connected by a path $u=u_{0}, u_{1}, \ldots, u_{n}=v$ in $\mathbf{N}^{n}$ with $u_{i+1}-u_{i} \in \pm \mathcal{B}$ if and only if $p^{u}-p^{v} \in I_{\mathcal{B}}$.

Diaconis, Eisenbud and Sturmfels [10] proposed analyzing the connectivity of the fibers of $\mathcal{B}$ using a primary decomposition of the ideal $I_{\mathcal{B}}$. In Section 2 we study the positive margins property and relate it to decompositions of $I_{\mathcal{B}}$. In particular, Lemma 2.5 gives a sufficient condition and a necessary condition for the positive margins property to hold. We also study a generalization of the positive margins property, which we call interior point property.

Our ideals $I_{\mathcal{B}}$ are conditional independence ideals, in addition to being motivated by the application to random walks. Primary decompositions of conditional independence ideals are interesting in their own right, since they reveal important information about the set of probability distributions that satisfy the conditional independence statements $[12,17,24,25,32]$. Moreover, it is interesting to know whether $I_{\mathcal{B}}$ is radical. Section 3 provides background on graphical models and conditional independence.

Section 4 studies the positive margins property of graphical models and radicality of their global Markov ideals. Both properties are preserved when forming the coned graph and when gluing graphs along cliques. In particular, decomposable graphs have both properties. 
From our results we deduce that, if all nodes are binary, then all global Markov ideals of graphs on five or fewer nodes are radical, and the complete bipartite graph $K_{2,3}$ is the only graph on five or fewer nodes which does not satisfy the original positive margins property while it does satisfy the interior point property. We also find graphical models without positive margins property, for any choice of matrix $A$, when the contingency table is sufficiently large (Theorem 4.12).

The graphical models of the $N$-cycle and the complete bipartite graph $K_{2, N-2}$ (with restrictions on the sizes of the contingency tables) are discussed in detail in Sections 5 and 6. We construct Markov bases and show that the global Markov ideals are radical by computing the primary decompositions.

Our results suggest a number of different directions for further research. First, in our analysis, we profited from the fact that all conditional independence ideals that we studied are radical. There do exist non-radical global Markov ideals, but we do not know how abundant those are. Second, our proofs of the positive margins property and interior point property for $\mathcal{B}$ depend on knowledge of a Markov basis for the lattice $\mathbf{Z B}$. It remains an open problem to develop proofs that do not depend on that extra knowledge.

2. Lattice walks, binomial ideals and positive margins. As Diaconis, Eisenbud, and Sturmfels [10] observed, the connectivity of the lattice walk induced by the moves in $\mathcal{B}$ can be analyzed by looking at a decomposition of the ideal $I_{\mathcal{B}}$. Indeed, suppose that $I_{\mathcal{B}}=\cap_{i} I_{i}$. Then $p^{u}-p^{v} \in I_{\mathcal{B}}$ if and only if $p^{u}-p^{v} \in I_{i}$ for all $i$. The following example demonstrates how to profit from this simple idea.

Example 2.1 (cf., [10, Example 1.2]). Which lattice points in $\mathbf{N}^{2}$ can be connected by the moves $\mathcal{B}=\{(2,-2),(3,-3)\}$ ? The solution can be read off from the decomposition:

$$
I_{\mathcal{B}}=\left\langle p_{1}^{2}-p_{2}^{2}, p_{1}^{3}-p_{2}^{3}\right\rangle=\left\langle p_{1}-p_{2}\right\rangle \cap\left\langle p_{1}^{2}, p_{2}^{2}\right\rangle .
$$

Now, $p^{a}-p^{b} \in\left\langle p_{1}-p_{2}\right\rangle$ if and only if $a_{1}+a_{2}=b_{1}+b_{2}$, while $p^{a}-p^{b} \in\left\langle p_{1}^{2}, p_{2}^{2}\right\rangle$ if and only if $\max \left\{a_{1}, a_{2}\right\} \geq 2$ and $\max \left\{b_{1}, b_{2}\right\} \geq 2$. Hence, $a$ and $b$ are connected by $\mathcal{B}$ if and only if $a_{1}+a_{2}=b_{1}+b_{2}$ and $\min \left\{\max \left\{a_{1}, a_{2}\right\}, \max \left\{b_{1}, b_{2}\right\}\right\} \geq 2$. 
The first decomposition that comes to mind is primary decomposition. If the ground field is algebraically closed, then, since $I_{\mathcal{B}}$ is binomial, there is a binomial primary decomposition $I_{\mathcal{B}}=\cap_{i} P_{i}$, where $P_{i}$ are generated by binomials. When the primary decomposition introduces new coefficients, then it is too fine to accurately reflect the combinatorics of $\mathcal{B}$-everything that matters in Proposition 1.2 are pure differences (i.e., binomials of the form $p^{u}-p^{v}$ ). In this case one should work with a mesoprimary decomposition of $I_{\mathcal{B}}[\mathbf{2 0}]$, the finest decomposition into unital binomial ideals (i.e., ideals generated by pure differences and monomials). In the examples studied in this paper all ideals $I_{\mathcal{B}}$ are radical, and the primary and mesoprimary decompositions agree.

The "most important" associated prime of $I_{\mathcal{B}}$, according to $[\mathbf{3 0}$, page $116]$, is the toric ideal

$$
I_{\mathbf{Z B}}=I_{\mathcal{B}}:\left(\prod_{i \in[n]} p_{i}\right)^{\infty}
$$

which is the only associated prime of $I_{\mathcal{B}}$ that does not contain variables. It equals the kernel of the ring homomorphism

$$
\begin{aligned}
\phi_{A}^{*}: \mathbf{k}\left[p_{i}: i=1, \ldots, n\right] & \longrightarrow \mathbf{k}\left[\theta_{j}, \theta_{j}^{-1}: j=1, \ldots, h\right], \\
p_{i} & \longrightarrow \prod_{j} \theta_{j}^{A_{j, i}}
\end{aligned}
$$

where $A$ is an integral matrix such that $\operatorname{ker}_{\mathbf{Z}} A=\mathbf{Z B}$. Equivalently, $I_{\mathbf{Z B}}=\left\langle p^{u}-p^{v}: u, v \in \mathbf{N}^{n}, A u=A v\right\rangle[\mathbf{2 9}]$. From this we obtain the following theorem.

Theorem 2.2 (Fundamental theorem of Markov bases [12, Theorem 1.3.6]). A set $\mathcal{B} \subseteq \operatorname{ker}_{\mathbf{Z}} A$ is a Markov basis if and only if $I_{\mathcal{B}}=I_{\mathbf{Z} \mathcal{B}}$.

The following is our basic definition.

Definition 2.3. Assume that $\mathcal{B}$ generates a saturated lattice $\mathbf{Z B}$, and let $A$ be a non-negative integer matrix such that $\mathbf{Z B} \subseteq \operatorname{ker}_{\mathbf{Z}} A$. Then $\mathcal{B}$ has the positive margins property (with respect to $A$ ) if $(A u)_{i}>0$ for all $i$ implies that the fiber of $u$ is connected. 
In most of the examples below, $\mathbf{Z B}=\operatorname{ker}_{\mathbf{Z}} A$. Still, the choice of matrix $A$ is crucial. In many situations there is a canonical choice, such as the marginal computing matrix in the case of graphical models (see Section 3). We can augment any matrix by adding rows which do not affect $\operatorname{ker}_{\mathbf{Z}} A$, but yield further nontrivial positivity conditions to check. A natural choice is to add all linear functionals corresponding to facets of the cone $\mathbf{R}_{\geq 0} A$ generated by the columns of $A$. In this case, the condition $(A u)_{i}>0$ for all $i$ says that $A u$ lies in the relative interior of the cone $\mathbf{R}_{\geq 0} A$.

Definition 2.4. Let $\mathcal{B}$ be a set of generators of the integer kernel $\operatorname{ker}_{\mathbf{Z}} A$ of the integer matrix $A$. Then $\mathcal{B}$ has the interior point property if it connects every fiber for which $A u$ lies in the relative interior of the cone $\mathbf{R}_{\geq 0} A$.

We now prove an algebraic criterion to decide the positive margins property. For any ideal $I \subseteq \mathbf{k}[p]$, let $m_{I}:=\prod\left\{p_{i} \notin I\right\}$ be the product of the variables not contained in $I$, and let $u_{I}$ be the exponent vector of $m_{I}$. We also need the product $\widehat{m}_{I}:=\prod\left\{p_{i}:\left(I: p_{i}\right)=I\right\}$ of all variables that are regular modulo $I$ and its exponent vector $\widehat{u}_{I}$. If $I$ is a prime or a radical cellular ideal, then $m_{I}=\widehat{m}_{I}$.

Lemma 2.5. Let $\mathcal{B}$ span a saturated sublattice of $\operatorname{ker}_{\mathbf{Z}} A$ for some non-negative integer matrix $A$. Let $I_{\mathcal{B}}=\left(\cap_{i=1}^{c} I_{i}\right) \cap I_{\mathbf{Z} \mathcal{B}}$ be a decomposition such that $I_{\mathbf{Z B}} \nsubseteq I_{i}$ for all $i$.

- If, for all $i=1, \ldots, c$, there exists $j$ such that $\left(A u_{I_{i}}\right)_{j}=0$, then $\mathcal{B}$ has the positive margins property with respect to $A$.

- If $\mathcal{B}$ has the positive margins property with respect to $A$, then for all $i=1, \ldots, c$, there exists $j$ such that $\left(A \widehat{u}_{I_{i}}\right)_{j}=0$.

Proof. For the first statement, suppose that $u, v \in \mathbf{N}^{n}$ lie in the same fiber but are not connected. Then $p^{u}-p^{v} \in I_{\mathbf{Z B}} \backslash I_{\mathcal{B}}$, and hence $p^{u}-p^{v} \notin I_{i}$ for some $i$. In particular, either $p^{u} \notin I_{i}$ or $p^{v} \notin I_{i}$. Assume that we are in the first case. Then $p^{u}$ is a divisor of $m_{I_{i}}^{a}$ for some integer $a$. Now, if there exists $j$ such that $\left(A u_{I_{i}}\right)_{j}=0$, then also $(A u)_{j}=0$, since $A$ is non-negative. This shows the first statement. 
For the second statement, suppose that $\left(A \widehat{u}_{I_{i}}\right)_{j}>0$ for some $i$ and all $j$. Let $p^{u}-p^{v}$ be a binomial in $I_{\mathbf{Z} \mathcal{B}} \backslash I_{i}$. Then $\left(A\left(u+\widehat{u}_{I_{i}}\right)\right)=$ $\left(A\left(v+\widehat{u}_{I_{i}}\right)\right)>0$, but since $\widehat{m}_{I_{i}}\left(p^{u}-p^{v}\right) \notin I_{i}$, the two vectors $u+\widehat{u}_{i}$ and $v+\widehat{u}_{i}$ are not connected by $\mathcal{B}$.

Note the asymmetry between the two directions, the first using $u_{I_{i}}$, the second $\widehat{u}_{I_{i}}$. If all $I_{i}$ are prime, then $m_{I_{i}}=\widehat{m}_{I_{i}}$. In this case, Lemma 2.5 gives an equivalent characterization of the positive margins property.

If the positive margins property is not satisfied, then one might still hope that the fibers are connected if the marginals are large enough. This is the case in Example 2.1. Unfortunately, if $I_{\mathcal{B}}$ is radical, then this is not true:

Lemma 2.6. Assume that $\mathcal{B}$ does not have the positive margins property with respect to the non-negative matrix $A$, and suppose that $I_{\mathcal{B}}$ is radical. For any $b>0$ there exist $u, v \in \mathbf{N}^{n}$ such that $(A u)_{j}=$ $(A v)_{j} \geq b$ for all $j$, but $p^{u}-p^{v} \notin I_{\mathcal{B}}$.

Proof. Let $I_{\mathcal{B}}=I_{\mathbf{Z} \mathcal{B}} \cap\left(\cap_{i} P_{i}\right)$ be the decomposition into minimal primes. By assumption and Lemma 2.5, for some $i$ the vector $u_{P_{i}}$ satisfies $\left(A u_{P_{i}}\right)_{j}>0$ for all $j$. For any binomial $p^{u}-p^{v} \in I_{\mathbf{Z} \mathcal{B}} \backslash P_{i}$, there exists a $c$ large enough such that the exponents satisfy $\left(A\left(u+c u_{P_{i}}\right)\right)_{j}=$ $\left(A\left(v+c u_{P_{i}}\right)\right)_{j} \geq b$ for all $j$. Since $P_{i}$ is prime, $m_{P_{i}}$ is regular and therefore $m_{P_{i}}^{c}\left(p^{u}-p^{v}\right) \notin P_{i}$. Hence, $u+c u_{i}$ and $v+c u_{i}$ are not connected.

Example 2.1 shows that the radicality assumption in Lemma 2.6 is necessary.

3. Graphical models and the global Markov statements. Let $V=[N]:=\{1, \ldots, N\}$ for some integer $N>1$. For each $v \in V$, let $X_{v}$ be a discrete random variable taking values in $\left[d_{v}\right], d_{v} \geq 2$. Let $d=\left(d_{v}\right)_{v \in V}$, and let $\mathcal{X}=\prod_{v \in V}\left[d_{v}\right]$. For any $W \subseteq V$, the random vector $X_{W}=\left(X_{v}\right)_{v \in W}$ takes values in $\mathcal{X}_{W}=\prod_{v \in W}\left[d_{v}\right]$. If $x \in \mathcal{X}$ and $W \subseteq V$, let $x_{W}:=\left(x_{v}\right)_{v \in W}$. With $h=|\mathcal{X}|$, denote by $\mathbf{R}^{h}:=\bigotimes_{v \in V} \mathbf{R}^{\frac{1}{d_{v}}}$ the space of real $d_{v_{1}} \times \cdots \times d_{v_{r}}$ arrays of the form 
$p=\left(p_{x}\right)_{x \in \mathcal{X}}$. Then $\mathbf{R}^{h}$ contains the probability simplex

$$
\Delta_{h-1}:=\left\{p \in \mathbf{R}^{h}: \sum_{x \in \mathcal{X}} p_{x}=1, p_{x} \geq 0 \quad \text { for all } x \in \mathcal{X}\right\} .
$$

Each $p \in \Delta_{h-1}$ represents a joint probability distribution of $\left(X_{v}\right)_{v \in V}$. The dependencies among $X_{1}, \ldots, X_{N}$ are often visualized by an undirected graph $G=(V, E)$. In this paper, all graphs are undirected and simple. There are two ways that such a graph can be interpreted as a statistical model, i.e., as a family of joint probability distributions. The first leads to the global Markov model, the second to the graphical model.

The global Markov model associates to $G$ a family of conditional independence statements among the random variables. Let $V=$ $A \cup B \cup C$ be a partition of $V$ (into disjoint possibly empty sets), and let $p \in \Delta_{h-1}$. We write $X_{A} \perp X_{B} \mid X_{C}$ and say that $X_{A}$ is independent of $X_{B}$ given $X_{C}$ if and only if

$$
p_{x_{A} x_{B} x_{C}} p_{x_{A}^{\prime} x_{B}^{\prime} x_{C}}-p_{x_{A} x_{B}^{\prime} x_{C}} p_{x_{A}^{\prime} x_{B} x_{C}}=0
$$

for all possible values $x_{A}, x_{A}^{\prime}, x_{B}, x_{B}^{\prime}, x_{C}$ of $X_{A}, X_{B}, X_{C}$, respectively. See [12] for an introduction to conditional independence from an algebraic point of view.

For each $x_{c} \in \mathcal{X}_{C}$, we construct a matrix $P^{A, B, x_{C}}$ of format $\left|\mathcal{X}_{A}\right| \times$ $\left|\mathcal{X}_{B}\right|$, with columns indexed by $\mathcal{X}_{A}$ and rows indexed by $\mathcal{X}_{B}$. The entry in the $x_{A}, x_{B}$ position of $P^{A, B, x_{C}}$ is the probability $p_{x_{A} x_{B} x_{C}}$. The conditional independence statement $X_{A} \perp X_{B} \mid X_{C}$ is equivalent to the condition that, for all $x_{C} \in \mathcal{X}_{C}, \operatorname{rank}\left(P^{A, B, x_{C}}\right) \leq 1$. If $C=\varnothing$ we get one matrix, and in general we get $\left|\mathcal{X}_{C}\right|$ matrices.

Let $I_{X_{A} \perp X_{B} \mid X_{C}}$ be the ideal in $\mathbf{R}\left[p_{x}: x \in \mathcal{X}\right]$ generated by the $2 \times 2$ minors of all the matrices $P^{A, B, x_{C}}$. If $\mathcal{C}$ is a collection of conditional independence statements, we let

$$
I_{\mathcal{C}}=\sum_{\left(X_{A} \perp X_{B} \mid X_{C}\right) \in \mathcal{C}} I_{X_{A} \perp X_{B} \mid X_{C}}
$$

To the graph $G$, we associate the global Markov statements:

$\operatorname{gl}(G)=\left\{X_{A} \perp X_{B} \mid X_{C}: C\right.$ separates $A$ and $B$ in $\left.G, A \cup B \cup C=V\right\}$. 
Separation means that every path in $G$ from some vertex $a \in A$ to some vertex $b \in B$ traverses some vertex $c \in C$. The global Markov model of $G$ is the intersection of $\Delta_{h-1}$, and the variety of $I_{\mathrm{gl}(G)}$, i.e., it consists of all joint probability distributions satisfying $\mathrm{gl}(G)$. Note that, while most statements in this paper are independent of the choice of the field $\mathbf{k}$, only the variety over the real numbers has a natural statistical interpretation. In general, conditional independence statements are defined for arbitrary subsets $A, B, C \subseteq V$, and the global Markov statements are defined without the requirement $A \cup B \cup C=V$. However, if $A, B, C \subseteq V$ are disjoint subsets such that $A \cup B \cup C \neq V$ and such that $C$ separates $A$ and $B$, then the statement $X_{A} \perp X_{B} \mid X_{C}$ is implied by the statements in $\mathrm{gl}(G)$, see [14, Lemma 7.10].

Graphical models are defined parametrically: Let $\mathcal{C}(G)$ be the set of cliques of $G$, where a clique is a set of vertices $W \subseteq V$ such that if $v_{1}, v_{2} \in W, v_{1} \neq v_{2}$, then $\left(v_{1}, v_{2}\right)$ is an edge of $G$. To each clique $C \in \mathcal{C}(G)$ and each $x_{C} \in \mathcal{X}_{C}$, associate a parameter $\theta_{x_{C}}^{C}$ (or an indeterminate, depending on the context). Let $\theta^{C}:=\left(\theta_{x_{C}}^{C}\right)_{x_{C} \in \mathcal{X}_{C}}$. The image of the polynomial map

$$
\phi_{G}: \bigoplus_{C \in \mathcal{C}(G)} \mathbf{R}^{d_{C}} \longrightarrow \mathbf{R}^{h}, \quad \phi_{G, x}\left(\theta^{C_{1}}, \ldots, \theta^{C_{r}}\right)=\prod_{C \in \mathcal{C}(G)} \theta_{x_{C}}^{C},
$$

intersected with the probability simplex $\Delta_{h-1}$ is the parametrized graphical model $\mathcal{M}_{G}^{*}$. In other words, $\mathcal{M}_{G}^{*}$ consists of all probability distributions $p$ whose components can be written as a product of the form $p_{x}=\prod_{W \in \mathcal{C}} f_{W}(x)$, where $f_{W}$ are nonnegative functions that only depend on $x_{v}$ for $v \in W$. See [21] for more about graphical models.

The map $\phi_{G}$ induces the ring homomorphism

$$
\begin{aligned}
\phi_{G}^{*}: \mathbf{R}\left[p_{x}: x \in \mathcal{X}\right] & \longrightarrow \mathbf{R}\left[\theta_{y_{C}}^{C}: C \in \mathcal{C}(G), y_{C} \in \mathcal{X}_{C}\right], \\
p_{x} & \longmapsto \prod_{C \in \mathcal{C}(G)} \theta_{x_{C}}^{C},
\end{aligned}
$$

and its kernel $I_{G}=\operatorname{ker} \phi_{G}^{*}$ is the vanishing ideal of the image. Then $\mathcal{M}_{G}=V\left(I_{G}\right) \cap \Delta_{h-1}$ is the closure of the parametrized graphical model $\mathcal{M}_{G}^{*}$. We call $\mathcal{M}_{G}$ the graphical model of $G$. Note that other authors use the term "graphical model" only for the set of strictly positive probability distributions in $\mathcal{M}_{G}$. 
The ring homomorphism $\phi_{G}^{*}$ is of the form (2.1); hence, $I_{G}$ is a toric ideal. The corresponding matrix $A_{G}$ has a natural interpretation: If $p$ is a joint probability distribution of $\left(X_{v}\right)_{v \in V}$, then the product $A_{G} p$ contains, as subvectors, the marginal distribution induced by $p$ on any clique of $G$. This collection of marginals is the G-marginals of $p$. The cone generated by the columns of $A_{G}$ is known as the marginal cone.

It is easy to check that the graphical model is a subset of the global Markov model. Moreover, the Hammersley-Clifford theorem [2] says that, if a probability distribution is strictly positive (that is, $p_{x}>0$ for all $x$ in the state space), then $p$ lies in the graphical model if and only if $p$ lies in the global Markov model. Algebraically, this theorem says that $I_{G}$ equals the toric component of $I_{\mathrm{gl}(G)}$.

In general, $I_{\mathrm{gl}(G)} \subsetneq I_{G}$, in which case, there may be probability distributions which satisfy the conditional independence statements $\mathrm{gl}(G)$, but are not in the closure of the graphical model. In fact, $I_{\mathrm{gl}(G)}=I_{G}$ if and only if $G$ is a chordal graph [15]. As suggested in [15] and [30, Chapter 8], the discrepancy between the two models can be analyzed using primary decomposition.

4. The positive margins property and graphical models. In this section we study which global Markov models have the positive margins property. Let $G$ be a graph with vertex set $V=[N]$, and let $d=\left(d_{v}\right)_{v \in V} \in \mathbf{N}^{N}$ with $d_{v} \geq 2$ for all $v$. We say that $(G, d)$ has the positive margins property, if the quadratic moves $\mathcal{B}_{\mathrm{gl}(G)}$ have the positive margins property with respect to the canonical matrix $A_{G}$, and $(G, d)$ has the interior point property if $\mathcal{B}_{\mathrm{gl}(G)}$ has the interior point property.

Our main tool is Lemma 2.5 which we translate here to graphical models. As all global Markov ideals with known primary decompositions are radical, we only formulate the radical case.

Lemma 4.1. Let $I_{\mathrm{gl}(G)}=\left(\cap_{i=1}^{c} P_{i}\right) \cap I_{G}$ be a decomposition into prime ideals such that $I_{G} \nsubseteq P_{i}$ for all $i$. Then $(G, d)$ has the positive margins property if and only if, for all $i=1, \ldots, c$, the $G$-margins of $u_{P_{i}}$ are not strictly positive. 
TABLE 1. Properties of binary graphical models for selected irreducible graphs.

\begin{tabular}{|l|c|c|c|c|}
\hline graph & pos. margins & interior point & $I_{\mathrm{gl}(G)}$ radical & \# of min. primes \\
\hline$C_{4}$ & yes & yes & yes & 9 \\
square-pyramid & yes & yes & yes & 81 \\
$G_{48}$ & yes & yes & yes & 201 \\
$K_{2,3}$ & no & yes & yes & 37 \\
$C_{5}$ & yes & yes & yes & 41 \\
\hline
\end{tabular}

Table 1 summarizes some of our computational results. We computed Markov bases with 4ti2 [1] and binomial primary decompositions using the package Binomials [18] in Macaulay2 [16]. Then we used the Macaulay2 package Polyhedra [3] to check the condition of Lemma 4.1 applied to the primary decomposition.

The binary graphical model of every graph on five or fewer vertices that is not mentioned in Table 1 satisfies the positive margins property, and the corresponding global Markov ideals are radical.

These results suggest two general questions:

- Is it true that, for any graphical model the ideal $I_{\mathrm{gl}(G)}$ is radical $[14]$ ?

- Does every graphical model have the interior point property?

The answers to both questions are negative in general. Example 4.9 discusses the binary CI ideal of $K_{3,3}$ which is not radical. Theorem 4.10 settles the second question.

Before discussing the graphs of Table 1, we treat reducible graphs. Note that all graphs on five or fewer vertices not contained in this table are either complete or decomposable, in the following sense:

Definition 4.2. A graph $G=(V, E)$ is reducible if there exist proper subsets $V_{1}, V_{2} \subset V$ such that $V_{1} \cap V_{2}$ is a clique, and such that $G$ is the union of the subgraphs $G_{1}$ and $G_{2}$ induced on $V_{1}$ and $V_{2}$. Moreover, $G$ is decomposable if $G_{1}$ and $G_{2}$ are complete or decomposable.

Lemma 4.3. Let $\mathbf{k}$ be algebraically closed. Assume that $G$ is reducible into $G_{1}=\left(V_{1}, E_{1}\right)$ and $G_{2}=\left(V_{2}, E_{2}\right)$. If both $I_{\mathrm{gl}\left(G_{1}\right)}$ and $I_{\mathrm{gl}\left(G_{2}\right)}$ are radical, then $I_{\mathrm{gl}(G)}$ is radical. 
Proof. This is [14, Corollary 7.13] together with the observation that the toric fiber product of prime ideals is a prime ideal.

Lemma 4.4. If $G$ is reducible into $G_{1}=\left(V_{1}, E_{1}\right)$ and $G_{2}=\left(V_{2}, E_{2}\right)$, and if both $\left(G_{1},\left(d_{v}\right)_{v \in V_{1}}\right)$ and $\left(G_{2},\left(d_{v}\right)_{v \in V_{2}}\right)$ have the positive margins property, then $(G, d)$ also has the positive margins property.

Proof. The proof is essentially the same as that of [31, Theorem 2.9], which shows how to obtain a Markov basis of $G$ from Markov bases of $G_{1}$ and $G_{2}$. The fact that we do not have Markov bases here is compensated by the fact that we do not want to connect all fibers, but just those fibers with positive margins. In order to apply the proof of [31, Theorem 2.9], two things need to be checked: (1) A fiber with positive $G$-margins restricts to fibers with positive $G_{1}$-margins and $G_{2^{-}}$ margins, respectively.

(2) When the construction that turns Markov bases of $G_{1}$ and $G_{2}$ into a Markov basis of $G$ is applied to $\mathcal{B}_{\mathrm{gl}\left(G_{1}\right)}$ and $\mathcal{B}_{\mathrm{gl}\left(G_{2}\right)}$, then the result is a subset of $\mathcal{B}_{\mathrm{gl}}(G)$. For brevity, we omit the details.

By Lemmas 4.3 and 4.4, decomposable graphs have the positive margins property and radical global Markov ideals (for all $d$ ). On four or fewer vertices, there is only one graph, the four-cycle $C_{4}$, which is neither complete nor decomposable. The following theorem is proved in Section 5.

Theorem 4.5. For $N \geq 4$, the binary $N$-cycle model has the positive margins property. Its global Markov ideal $I_{\mathrm{gl}\left(C_{N}\right)}$ is radical.

On five vertices, there are five irreducible graphs: The complete graph (which trivially has the positive margins property), the fivecycle $C_{5}$ (covered by Theorem 4.5 ), the complete bipartite graph $K_{2,3}$, the square pyramid, and the graph $G_{48}$ (see Figure 1 ; the name $G_{48}$ comes from [27]). The complete bipartite graph $K_{2,3}$ is treated in the following theorem, proved at the end of Section 6 . 

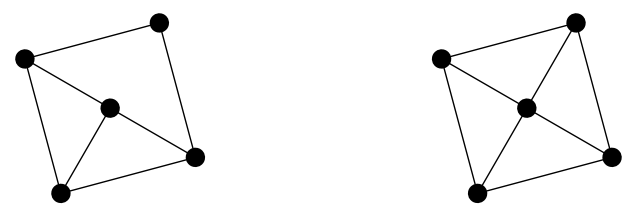

FIGURE 1. $G_{48}$ and the square pyramid.

Theorem 4.6. For $N \geq 4$, the complete bipartite graph $K_{2, N-2}$, where $d_{v}=2$ for the first group of 2 nodes, has the interior point property. It has the positive margins property if and only if $N=4$. Its global Markov ideal is radical for all $N \geq 4$.

We next discuss the pyramid. To obtain a more general result the following definition is needed: For any graph $G=(V, E)$ with vertex set $V=[N]$, the cone over $G$ is the graph $\widehat{G}=(\widehat{V}, \widehat{E})$ with $\widehat{V}=V \cup\{0\}$ and $\widehat{E}=E \cup\{(0, i): i \in[N]\}$.

Lemma 4.7. Assume that $\mathbf{k}$ is a perfect field. If $I_{\mathrm{gl}(G)}$ is radical for some $d \in \mathbf{N}^{V}$, then $I_{\mathrm{gl}(\hat{G})}$ is radical for all $\widehat{d} \in \mathbf{N}^{\hat{V}}$ with $\widehat{d}_{v}=d_{v}$ for all $v \in V$.

Proof. Let $\widehat{\mathcal{X}}=\left[d_{0}\right] \times \mathcal{X}$. For any polynomial $f \in \mathbf{k}\left[p_{x}: x \in \mathcal{X}\right]$, denote by $\widehat{f}_{i}$ the polynomial in $\mathbf{k}\left[p_{y}: y \in \widehat{\mathcal{X}}\right]$ where each variable $p_{x}$, $x \in \mathcal{X}$, has been replaced by $p_{i x}$. Let $I_{i}$ be the ideal generated by the polynomials $\widehat{f}_{i}$ for all $f \in I_{\mathrm{gl}(G)}$. The equality

$$
\operatorname{gl}(\widehat{G})=\left\{X_{A} \perp X_{B}\left|X_{C} \cup X_{0}: X_{A} \perp X_{B}\right| X_{C} \in \operatorname{gl}(G)\right\}
$$

implies $I_{\operatorname{gl}(\hat{G})}=I_{1}+\cdots+I_{d_{0}}$. The ideals $I_{1}, \ldots, I_{d_{0}}$ are radical, since $I_{\mathrm{gl}(G)}$ is radical, and $I_{1}, \ldots, I_{d_{0}}$ are generated by polynomials in disjoint sets of variables. To show that their sum is also radical it suffices to show that the tensor product of reducible rings is again reducible. This is true if the field $\mathbf{k}$ is perfect by $[4$, Chapter 5 , Section $15]$.

Lemma 4.8. If $(G, d)$ has the positive margins property, then $(\widehat{G}, \widehat{d})$ also has the positive margins property, where $\widehat{d}_{v}=d_{v}$ for $v \in[N]$ and $\widehat{d}_{0}$ is arbitrary. 
Proof. Any contingency table $\widehat{u}$ for $\widehat{G}$ can be seen as a family $\left(u^{(i)}\right)_{i \in\left[\hat{d}_{0}\right]}$ of contingency tables for $G$. If $\widehat{u}$ has positive $\widehat{G}$-margins, then each $u^{(i)}$ has positive $G$-margins. Now $\widehat{u}$ and $\widehat{v}$ have the same $\widehat{G}$ margins if and only if $u^{(i)}$ and $v^{(i)}$ have the same $G$-margins for all $i$. Hence, if $\widehat{u}$ and $\widehat{v}$ have the same positive $\widehat{G}$-margins, then $u^{(i)}$ and $v^{(i)}$ are connected by quadratic moves for all $i$, and the same moves can be used to connect $\widehat{u}$ and $\widehat{v}$.

It remains to discuss $G_{48}$. It is easy to see that the binary model for this graph is equal to the model of $K_{2,2}$ with $d=(2,2,2,4)$ and therefore covered by Theorem $4.6-G_{48}$ has the positive margins property, and its global Markov ideal is radical.

Next, we give an example of a global Markov ideal that is not radical.

Example 4.9. Consider the graph $K_{3,3}$, and let $d_{v}=2$ for all vertices $v \in K_{3,3}$. The global Markov ideal $I_{\mathrm{gl}\left(K_{3,3}\right)}$ is contained in a polynomial ring with 64 indeterminates. It is generated by 144 quadrics corresponding to the six CI statements $X_{i} \perp X_{j k} \mid X_{456}$ and $X_{i} \perp X_{j k} \mid X_{123}$, where $\{i\} \cup\{j k\}$ runs through the non-trivial bipartitions of $\{1,2,3\}$ and $\{4,5,6\}$, respectively.

The global Markov ideal $I_{\mathrm{gl}\left(K_{3,3}\right)}$ is complicated enough that Buchberger's algorithm for Gröbner basis computation does not terminate within a reasonable time. On the other hand, a Gröbner basis of the graphical model $I_{K_{3,3}}$ can be computed using 4 ti2 [1]. This is another example of the fact that toric ideals are less complex than arbitrary binomial ideals [28].

In view of these complications, the classical tools of computer algebra do not work for this example, as they depend on Gröbner bases. However, we can use Proposition 1.1: containment of a binomial in a pure difference ideal can be checked by analyzing the connected components of a graph. We implemented this idea in a C++-library that can test whether two exponent vectors lie in the same connected component by enumerating their connected components via breadthfirst search. The $\mathrm{C}++$-source code of our library is available on the internet under the GPL licence [19]. The directory examples contains code for $K_{3,3}$, and other graphs, which generates the connected components and constructs a path in the case of connectivity. 
To disprove radicality, it suffices to find a binomial $p^{u}-p^{v} \in$ $I_{K_{3,3}} \backslash I_{\mathrm{gl}\left(K_{3,3}\right)}$ (for example, a degree four Markov move) and a square-free monomial $p^{w}$ such that $p^{w}\left(p^{u}-p^{v}\right) \notin I_{\mathrm{gl}\left(K_{3,3}\right)}$ while $p^{2 w}\left(p^{u}-p^{v}\right) \in I_{\mathrm{gl}\left(K_{3,3}\right)}$. Checking the degree four Markov moves $p^{u}-p^{v}$ and monomials of degree two, our program found the following witness: Let

$p^{u}-p^{v}:=p_{121 \mid 222} p_{212 \mid 212} p_{122 \mid 112} p_{222 \mid 122}-p_{221 \mid 222} p_{112 \mid 212} p_{222 \mid 112} p_{122 \mid 122}$, and let $p^{w}:=p_{111 \mid 111} p_{221 \mid 111}$ (the vertical bar $\mid$ separates the states of the two groups of nodes in $\left.K_{3,3}\right)$. Then $p^{u} p^{w}$ and $p^{v} p^{w}$ are not connected by $\operatorname{gl}\left(K_{3,3}\right)$, but $p^{u} p^{2 w}$ and $p^{v} p^{2 w}$ are connected. The connected components of $p^{u+w}$ and $p^{v+w}$ consist of 18 monomials each, while that of $p^{u} p^{2 w}$ and $p^{v} p^{2 w}$ consists of 90 monomials.

We now construct examples of graphical models that do not have the interior points property (and, hence, cannot have any positive margins property). Remember that a graph $G$ is triangle-free if it does not contain a cycle of length three, and a graph is two-connected if it remains connected when a single node is eliminated.

Theorem 4.10. Let $G$ be a two-connected triangle-free graph with $N$ vertices, and let $p \geq N-1$ be a prime power. If $d_{a}=p$ for all $a \in[N]$, then $(G, d)$ does not have the interior point property.

Before proving the theorem, we first give an explicit example.

Example 4.11. Consider the four-cycle $C_{4}$ with $d=(3,3,3,3)$, and let

$u=e_{1111}+e_{1222}+e_{1333}+e_{2123}+e_{2231}+e_{2312}+e_{3132}+e_{3213}+e_{3321}$.

The marginal vector $A_{C_{4}} u$ of $u$ lies in the interior of the marginal cone, and many other vectors with the same marginals can be constructed by applying elements of the symmetry group $(\mathbf{Z} / 3 \mathbf{Z})^{4}$. At the same time, no quadratic move can be applied to $u$.

The combinatorially inclined reader may have observed two orthogonal Latin squares of order three in the last two indices of the elements 
contributing to $u$. Recall that a Latin square of order $d$ is a $(d \times d)$ matrix $L$ with entries in $[d]$ such that each number in $[d]$ appears exactly once in each row and in each column. Two Latin squares $L, L^{\prime}$ are orthogonal if $\left(L_{i, j}, L_{i, j}^{\prime}\right)=\left(L_{k, l}, L_{k, l}^{\prime}\right)$ implies $i=k$ and $j=l$. For general $d$, the number of mutually orthogonal Latin squares of order $d$ is not known. The following is known:

1. There are at least two orthogonal Latin squares of order $d$, unless $d \in\{1,2,6\}$.

2. There are at most $(d-1)$ orthogonal Latin squares of order $d$.

3 . If $d$ is a power of a prime, then there are precisely $(d-1)$ orthogonal Latin squares of order $d$.

See [8] for an introduction to Latin squares. Theorem 4.10 is a corollary to these facts and the following theorem.

Theorem 4.12. Let $G$ be a two-connected triangle-free graph with $N$ vertices. If there exist $N-2$ mutually orthogonal Latin squares of order $d_{0} \geq 2$, then $\left(G,\left(d_{0}, d_{0}, \ldots, d_{0}\right)\right)$ does not satisfy the interior point property.

Proof. Let $L^{(1)}, \ldots, L^{(N-2)}$ be mutually orthogonal Latin squares of order $d_{0}$, and let

$$
\mathcal{L}=\left\{\left(i, j, L_{i, j}^{(1)}, \ldots, L_{i, j}^{(N-2)}\right): i, j \in\left[d_{0}\right]\right\} \subset \mathcal{X}=\left[d_{0}\right]^{N} .
$$

The set $\mathcal{L}$ has the property that, for every pair $a, b \in[N], a \neq b$, one has

$$
\left\{\left(l_{a}, l_{b}\right): l \in \mathcal{L}\right\}=\left[d_{0}\right]^{2} .
$$

Since $G$ is triangle-free, all $G$-margins are 2-way margins. The vector $u(\mathcal{L})$ defined via

$$
u(\mathcal{L})(l)= \begin{cases}1 & \text { if } l \in \mathcal{L}, \\ 0 & \text { otherwise }\end{cases}
$$

has the following property: all entries in all its 2-way margins are ones. The group $\mathfrak{S}_{d_{0}}^{N}\left(N\right.$ th direct power of the symmetric group $\mathfrak{S}_{d_{0}}$ of $\left.\left[d_{0}\right]\right)$ acts on $\mathcal{X}$ by permuting each factor. This action induces an action on the marginal cone that is transitive on the extreme rays. Under this action, the margins of $u(\mathcal{L})$ are invariant, which implies that $A_{G} u(\mathcal{L})$ lies in the interior of this cone and, in particular, is not on any facet. 


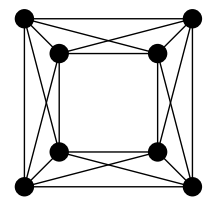

FIGURE 2. Splitting all vertices of $C_{4}$.

On the other hand, it is not possible to apply any quadratic global Markov move to the table $u(\mathcal{L})$. Indeed, since $G$ is two connected, any quadratic move $v$ corresponds to a statement $X_{A} \perp X_{B} \mid X_{C}$, where the separator $C$ contains at least two distinct elements $i, j$. Hence, $v$ can only be applied to tables where some entry in the $(i, j)$-marginal is two. Therefore, $u(\mathcal{L})$ is isolated in its fiber. On the other hand, the symmetric group action on tables sends $u(\mathcal{L})$ to other points in its fiber, so that the fiber is disconnected.

Example 4.13 (A binary grapical model without interior point property). We can use Theorem 4.12 to show that not all graphs with binary nodes have the interior point property. First, $C_{4}$ with $d=(4,4,4,4)$ does not have the interior point property, since there exists a pair of orthogonal Latin squares of order four. We define a graph $G$ by splitting every vertex of $C_{4}$ into an edge as in Figure 2 .

It can be seen that the binary model of $G$ is equivalent to that of $C_{4}$ with $d=(4,4,4,4)$ in the sense that the ideals $I_{C_{4}}$ and $I_{\mathrm{gl}\left(C_{4}\right)}$ are related to the ideals $I_{G}$ and $I_{\mathrm{gl}(G)}$ via a renaming of the coordinates.

All examples where we could prove the interior point property involve graphs where the toric ideal $I_{G}$ is generated in degree at most four, and our proofs of the primary decomposition also depend on this fact.

Question 4.14. If $I_{G}$ is generated in degree at most four, does this imply that $(G, d)$ has the interior point property?

There are five graphs $G$ on $I_{G}$ such that $I_{G}$ (with $d_{v}=2$ for all $v$ ) is not generated in degree four; and, in this case, $I_{G}$ is generated in degree six [26]. Among these graphs, $K_{3,3}$ and $G_{154}$ are the only triangle-free 

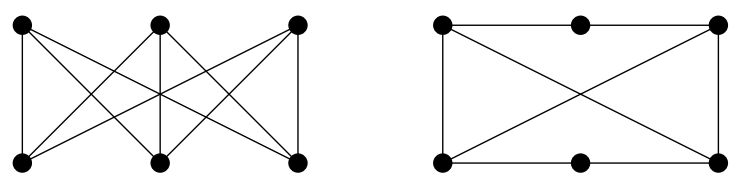

FIGURE 3. $K_{3,3}$ and $G_{154}$.

graphs. It is a challenging problem to compute primary decompositions of $I_{\mathrm{gl}(G)}$ for these two graphs. By Example 4.9, $I_{\mathrm{gl}\left(K_{3,3}\right)}$ is not radical. The same method did not allow us to disprove radicality of $I_{\mathrm{gl}\left(G_{154}\right)}$. Note that $G_{154}$ can be obtained from $K_{3,3}$ by deleting an edge.

Theorem 4.10 proves that, for any two connected triangle-free graph, if the cardinalities $d=\left(d_{v}\right)_{v \in V}$ are increased to all coincide with the same prime power, then this model does not have the interior point property. We conjecture that this generalizes to many other graphs, i.e., the situation should not improve when the numbers $d_{v}$ are increased. A similar phenomenon occurs with Markov bases. For instance, in the no-three-way interaction model, the Markov basis becomes arbitrarily complicated as two of the $d_{v}$ diverge $[\mathbf{2 2}]$.

Conjecture 4.15. Assume that $(G, d)$ does not have the positive margins (or interior point) property. If $d_{v}^{\prime} \geq d_{v}$ for all $v \in V$, then $\left(G, d^{\prime}\right)$ does not have the positive margins (or interior point) property either.

5. Binary $N$-cycle models. In this section, we study the binary model of the $N$-cycle $C_{N}$. We find a Markov basis (Theorem 5.1) and compute a prime decomposition, showing that $I_{\mathrm{gl}\left(C_{N}\right)}$ is radical (Theorem 5.6). We then use this decomposition to prove the positive margins property (Theorem 4.5).

We first describe a Markov basis of the toric ideal $I_{C_{N}}$. A Markov basis for this model was already presented in [9]. Here, we construct a smaller Markov basis in order to simplify our proofs of the primary decomposition. We use tableau notation to denote monomials and 
binomials in the polynomial ring $\mathbf{k}\left[p_{x}: x \in \mathcal{X}\right]$. The monomial $p_{x_{1,1} x_{1,2} \cdots x_{1, N}} \cdots p_{x_{t, t} x_{t, 2} \cdots x_{t, N}}$ is represented by the following tableau with $t$ rows:

$$
\left[\begin{array}{cccc}
x_{1,1} & x_{1,2} & \cdots & x_{1, N} \\
\vdots & \vdots & \vdots & \\
x_{t, t} & x_{t, 2} & \cdots & x_{t, N}
\end{array}\right] .
$$

This notation greatly facilitates computations since applying moves to a monomial merely corresponds to manipulating the entries of tableau according to rules encoded by the moves. Tableau calculations are widely used in algebraic statistics, see for example [12].

The cyclic group of order $N$ acts on $C_{N}$ by rotations, inducing an action on tableaux. For example, the generator of this symmetry group moves the rightmost column to the left, and all other columns move one step to the right. The Markov basis that we construct is invariant under this symmetry.

Let $\mathcal{B}_{2}$ be the set of quadrics that are, up to cyclic symmetry, of the form

$$
\begin{gathered}
{\left[\begin{array}{cccc}
a & A & b & B \\
a & A^{\prime} & b & B^{\prime}
\end{array}\right]-\left[\begin{array}{cccc}
a & A^{\prime} & b & B \\
a & A & b & B^{\prime}
\end{array}\right],} \\
a, b \in[2], \quad A, A^{\prime} \in[2]^{l}, \quad B, B^{\prime} \in[2]^{N-l-2}, \\
A \neq A^{\prime}, B \neq B^{\prime}, \quad 0<l<N-2 .
\end{gathered}
$$

The quadrics in $\mathcal{B}_{2}$ correspond to conditional independence statements of the form $\left\{X_{k+1}, \ldots, X_{l-1}\right\} \perp\left\{X_{l+1}, \ldots, X_{k-1}\right\} \mid\left\{X_{k}, X_{l}\right\}$ with $k$ and $l$ non-adjacent (here, indices are considered modulo $N$ ). Note that $\mathrm{gl}\left(C_{N}\right)$ contains further statements; but, their quadrics are contained in the ideal generated by those in $\mathcal{B}_{2}$.

To each binary state $K \in \mathcal{X}$ there is a unique opposite state $\bar{K} \in \mathcal{X}$, defined by switching 1 and 2 in each component. Let $\mathcal{B}_{4}$ be the set of quartics that are, up to cyclic symmetry, of the form 


$$
\left[\begin{array}{ccc}
A & \frac{B}{A} & C \\
\bar{A} & \bar{B} & \bar{C} \\
\bar{A} & \bar{B} & \bar{C} \\
\bar{B} & C
\end{array}\right]-\left[\begin{array}{ccc}
A & B & \bar{C} \\
\bar{A} & \bar{B} & C \\
\bar{A} & \bar{B} & \bar{C} \\
\bar{A} & \bar{B} & \bar{C}
\end{array}\right],
$$

$$
\begin{gathered}
A \in[2]^{k}, \quad B \in[2]^{l-k}, \quad C \in[2]^{N-l}, \\
0<k<l<N .
\end{gathered}
$$

Theorem 5.1. For $N \geq 4$, the set $\mathcal{B}_{2} \cup \mathcal{B}_{4}$ is a Markov basis of the binary graphical model of the $N$-cycle.

Remark 5.2. For $N=3$, the cycle is a complete graph, and therefore $I_{G}=I_{\mathrm{gl}(G)}=0$. The single generator contained in $\mathcal{B}_{4}$ defines another interesting statistical model: the no-three-way interaction model. It is the hierarchical model of the graph $C_{3}$, considered as a one-dimensional simplicial complex (see [12]). Such models were named graph models in [9], in order to distinguish them from graphical models. For $N \geq 4$, all cliques of the $N$-cycle are edges, and therefore the graphical model agrees with the graph model.

Proof of Theorem 5.1. We prove that $\mathcal{B}_{2} \cup \mathcal{B}_{4}$ is actually a Markov basis of the graph model of $C_{N}$ for all $N \geq 1$. We use induction on $N$. For $N<3$, both $\mathcal{B}_{2}$ and $\mathcal{B}_{4}$ are empty, and the graph model contains all probability distributions. For $N=3$, the set $\mathcal{B}_{2}$ is empty, while $\mathcal{B}_{4}$ contains only the defining quartic of the binary graph model of $C_{3}$.

The $N$-cycle is a codimension-one toric fiber product of a chain of length $(N-1)$ with a chain of length three (see $[\mathbf{1 4}]$ ). Since these chains are decomposable graphs, the Markov bases of these chains consist of quadratic moves, corresponding to conditional independence statements. These Markov bases are slow-varying, in the sense of [14], and gluing them yields moves in $\mathcal{B}_{2}$. By [14, Theorem 5.10], in order to obtain a Markov basis of the $N$-cycle, we need to add further quadrics (which belong to $\mathcal{B}_{2}$ ) and a Markov basis of the corresponding codimension-zero toric fiber product, which is the toric fiber product 
of an $(N-1)$-cycle with the graph model of the 3 -cycle. By induction, we know the Markov bases of these smaller cycles, and by [14, Theorem 5.4], we need to lift these Markov bases (and add some further quadrics that belong to $\mathcal{B}_{2}$ ). A lift of a quadric gives again a quadric from $\mathcal{B}_{2}$, and hence it suffices to consider the quartics.

We first show that the ideal $I_{\mathcal{B}_{2} \cup \mathcal{B}_{4}}$ contains all tableaux of the form

$$
\left[\begin{array}{llll}
1 & 1 & 1 & A \\
1 & 2 & 2 & B \\
2 & 1 & 2 & C \\
2 & 2 & 1 & D
\end{array}\right]-\left[\begin{array}{llll}
1 & 2 & 1 & A \\
1 & 1 & 2 & B \\
2 & 2 & 2 & C \\
2 & 1 & 1 & D
\end{array}\right]
$$

where each entry is a $\{1,2\}$-string of length at least one. Suppose that there is a column $k$ such that $A_{k}=C_{k}$. Without loss of generality, assume $A_{k}=C_{k}=1$. Decompose the strings $A, B, C, D$ into substrings, such that $A=\left(A_{l} A_{k} A_{r}\right)$, and so on. The tableau calculation

$$
\left[\begin{array}{llllll}
\mathbf{1} & \mathbf{1} & \mathbf{1} & A_{l} & 1 & A_{r} \\
\mathbf{1} & \mathbf{2} & \mathbf{2} & B_{l} & b & B_{r} \\
\mathbf{2} & \mathbf{1} & \mathbf{2} & C_{l} & 1 & C_{r} \\
\mathbf{2} & \mathbf{2} & \mathbf{1} & D_{l} & d & D_{r}
\end{array}{ }^{*} *\left[\begin{array}{cccccc}
\mathbf{1} & \mathbf{1} & \mathbf{2} & C_{l} & 1 & A_{r} \\
\mathbf{1} & \mathbf{2} & \mathbf{2} & B_{l} & b & B_{r} \\
\mathbf{2} & \mathbf{1} & \mathbf{1} & A_{l} & 1 & C_{r} \\
\mathbf{2} & \mathbf{2} & \mathbf{1} & D_{l} & d & D_{r}
\end{array}\right]+\begin{array}{c}
* \\
* \\
+ \\
+
\end{array}\right.
$$

$$
\longrightarrow\left[\begin{array}{llllll}
\mathbf{1} & \mathbf{2} & \mathbf{2} & C_{l} & 1 & A_{r} \\
\mathbf{1} & \mathbf{1} & \mathbf{2} & B_{l} & b & B_{r} \\
\mathbf{2} & \mathbf{2} & \mathbf{1} & A_{l} & 1 & C_{r} \\
\mathbf{2} & \mathbf{1} & \mathbf{1} & D_{l} & d & D_{r}
\end{array}\right] * *\left[\begin{array}{cccccc}
\mathbf{1} & \mathbf{2} & \mathbf{1} & A_{l} & 1 & A_{r} \\
\mathbf{1} & \mathbf{1} & \mathbf{2} & B_{l} & b & B_{r} \\
\mathbf{2} & \mathbf{2} & \mathbf{2} & C_{l} & 1 & C_{r} \\
\mathbf{2} & \mathbf{1} & \mathbf{1} & D_{l} & d & D_{r}
\end{array}\right]
$$

shows that this move actually lies in the ideal generated by the quadrics. Here, $*$ and + mark the rows to which a quadric has been applied. By symmetry, the same holds true if $B_{k}=D_{k}$ for some $k$. If, in the tableau (5.3), $A_{k} \neq C_{k}$ and $B_{k} \neq D_{k}$ for all $k$, then $C=\bar{A}$ and $D=\bar{B}$, and the move is of the form

$$
\left[\begin{array}{llll}
1 & 1 & 1 & A \\
1 & 2 & 2 & B \\
2 & 1 & 2 & \frac{A}{B} \\
2 & 2 & 1 & B
\end{array}\right]-\left[\begin{array}{cccc}
1 & 2 & 1 & A \\
1 & 1 & 2 & \frac{B}{A} \\
2 & 2 & 2 & \frac{A}{B} \\
2 & 1 & 1 & .
\end{array}\right]
$$

Hence, invoking the symmetry and exchanging $1 \leftrightarrow 2$ in some columns of the last block, we may assume that any column in the last block 
agrees with a column from either the first or the third block. If $\left[\begin{array}{l}A \\ B\end{array}\right]=\left[\begin{array}{ll}\mathbf{1} & 1 \\ \mathbf{2} & \mathbf{1}\end{array}\right]$, then the lift belongs to $\mathcal{B}_{4}$. Otherwise, using a rotation of the cycle, the move can be brought into the form

$$
\left[\begin{array}{llllll}
\mathbf{1} & \mathbf{1} & \mathbf{1} & 1 & A & 1 \\
\mathbf{1} & \mathbf{2} & \mathbf{2} & 1 & B & 2 \\
\mathbf{2} & \mathbf{1} & \mathbf{2} & 2 & \bar{A} & 2 \\
\mathbf{2} & \mathbf{2} & \mathbf{1} & 2 & \bar{B} & 1
\end{array}\right]-\left[\begin{array}{cccccc}
\mathbf{1} & \mathbf{2} & \mathbf{1} & 1 & A & 1 \\
\mathbf{1} & \mathbf{1} & \mathbf{2} & 1 & \frac{B}{2} & 2 \\
\mathbf{2} & \mathbf{2} & \mathbf{2} & 2 & \bar{A} & 2 \\
\mathbf{2} & \mathbf{1} & \mathbf{1} & 2 & \bar{B} & 1
\end{array}\right] .
$$

Applying quadrics to the first two rows transforms this into the move

$$
\left[\begin{array}{llllll}
\mathbf{1} & \mathbf{1} & \mathbf{1} & 1 & B & 2 \\
\mathbf{1} & \mathbf{2} & \mathbf{2} & 1 & A & 1 \\
\mathbf{2} & \mathbf{1} & \mathbf{2} & 2 & \bar{A} & 2 \\
\mathbf{2} & \mathbf{2} & \mathbf{1} & 2 & \bar{B} & 1
\end{array}\right]-\left[\begin{array}{cccccc}
\mathbf{1} & \mathbf{2} & \mathbf{1} & 1 & B & 2 \\
\mathbf{1} & \mathbf{1} & \mathbf{2} & 1 & \frac{A}{1} \\
\mathbf{2} & \mathbf{2} & \mathbf{2} & 2 & \bar{A} & 2 \\
\mathbf{2} & \mathbf{1} & \mathbf{1} & 2 & \bar{B} & 1
\end{array}\right] .
$$

In this move, the first and third entries of the last column agree, and, by the argument given above, it is a combination of quadrics. Now the theorem follows from the observation that, up to symmetry, any lifted quartic is of form (5.3).

For any quartic $f$ of form (5.2) let

$$
P_{f}=\left\langle p_{i}: p_{i} \text { divides neither } f^{+} \text {nor } f^{-}\right\rangle .
$$

Lemma 5.3. The ideals $P_{f}$ are prime ideals containing $I_{\mathrm{gl}\left(C_{N}\right)}$.

Proof. Clearly, $P_{f}$ is a monomial prime ideal. Each generator of $I_{\mathrm{gl}\left(C_{N}\right)}$ is of the form (5.1). If the left term $\left[\begin{array}{cccc}a & A & b & B \\ a & A^{\prime} & b & B^{\prime}\end{array}\right]$ is not contained in $P_{f}$, then it divides $f^{+} f^{-}$. In this case either $A=A^{\prime}$ or $B=B^{\prime}$, and so both terms in (5.1) agree. Hence, $P_{f}$ contains all generators of $I_{\mathrm{gl}\left(C_{N}\right)}$.

Proposition 5.4. The minimal primes of $I_{\mathrm{gl}\left(C_{N}\right)}$ are precisely the toric ideal $I_{C_{N}}$ and the monomial ideals $P_{f}$. 
The proof of Proposition 5.4 makes use of the following lemma.

Lemma 5.5. Let $f=f^{+}-f^{-} \in \mathcal{B}_{4}$ be a quartic generator of $I_{C_{N}}$. If the variable $p_{i}$ divides neither $f^{+}$nor $f^{-}$, then $p_{i} f \in I_{\mathrm{gl}\left(C_{N}\right)}$.

Proof. We have to show that $p_{i} f$ is a combination of quadrics coming from conditional independence statements of the $N$-cycle. Up to symmetry, $p_{i} f$ is of the form

$$
\left[\begin{array}{ccc}
1 & 1 & 1 \\
1 & 2 & 2 \\
2 & 1 & 2 \\
2 & 2 & 1 \\
K & L & M
\end{array}\right]-\left[\begin{array}{ccc}
2 & 1 & 1 \\
1 & 2 & 1 \\
1 & 1 & 2 \\
2 & 2 & 2 \\
K & L & M
\end{array}\right]
$$

where $p_{i}=p_{K L M}$. We now transform $p_{i} f$ into another binomial $p_{j} \tilde{f}$ of total degree five using quadrics. Then $p_{j} \tilde{f}$ belongs to the toric ideal $I_{C_{N}}$, and hence $\widetilde{f} \in I_{C_{N}}$. Since $p_{j} \neq p_{i}$ the multidegree of $\widetilde{f}$ is not the multidegree of any quartic in $\mathcal{B}_{4}$. Therefore, $\tilde{f}$ must be a combination of quadrics, and we are done.

Using symmetry we may assume that $K, L, M$ all contain at least one 1, i.e., $K L M=K_{1} 1 K_{2} L_{1} 1 L_{2} M_{1} 1 M_{2}$. The tableau calculation

$$
\begin{aligned}
& {\left[\begin{array}{lll}
\mathbf{2} & \mathbf{1} & \mathbf{1} \\
\mathbf{1} & \mathbf{2} & \mathbf{1} \\
\mathbf{1} & \mathbf{1} & \mathbf{2} \\
\mathbf{2} & \mathbf{2} & \mathbf{2} \\
K & L & M
\end{array}\right]_{*} *\left[\begin{array}{ccccccc}
\mathbf{2} & 2 & \mathbf{2} & 1 & \mathbf{1} & 1 & \mathbf{1} \\
\mathbf{1} & 1 & \mathbf{1} & 2 & \mathbf{2} & 2 & \mathbf{1} \\
\mathbf{1} & 1 & K_{2} & L_{1} & \mathbf{1} & 1 & \mathbf{2} \\
\mathbf{2} & 2 & \mathbf{2} & 2 & \mathbf{2} & 2 & \mathbf{2} \\
K_{1} & 1 & \mathbf{1} & 1 & \mathbf{1} & L_{2} & M
\end{array}\right] *} \\
& \longrightarrow\left[\begin{array}{ccccccccc}
\mathbf{2} & 2 & \mathbf{2} & \mathbf{1} & 1 & \mathbf{1} & \mathbf{1} & 1 & \mathbf{1} \\
K_{1} & 1 & \mathbf{1} & \mathbf{2} & 2 & \mathbf{2} & \mathbf{1} & 1 & M_{2} \\
\mathbf{1} & 1 & K_{2} & L_{1} & 1 & \mathbf{1} & \mathbf{2} & 2 & \mathbf{2} \\
\mathbf{2} & 2 & \mathbf{2} & \mathbf{2} & 2 & \mathbf{2} & \mathbf{2} & 2 & \mathbf{2} \\
\mathbf{1} & 1 & \mathbf{1} & \mathbf{1} & 1 & L_{2} & M_{1} 1 & \mathbf{1}
\end{array}\right]_{*}^{*}
\end{aligned}
$$

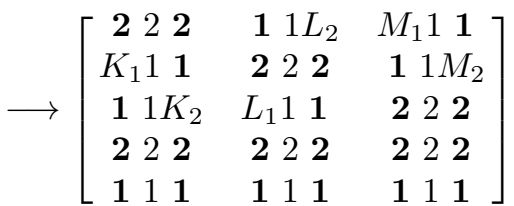


shows how to transform the second term of $p_{i} f$ such that the resulting binomial is of the form $p_{\mathbf{1 1 1}} \widetilde{f}$.

Proof of Proposition 5.4. Let $p \in V\left(I_{\mathrm{gl}\left(C_{N}\right)}\right) \backslash V\left(I_{C_{N}}\right)$. Then there is a quartic $f \in \mathcal{B}_{4}$ such that $f(p) \neq 0$. Lemma 5.5 implies that $p_{K}=0$ for all $K$ such that $p_{K}$ does not divide $f^{+} f^{-}$, and hence $p \in V\left(P_{f}\right)$. Clearly, the ideals $P_{f}$ are all distinct. By symmetry, they are all minimal primes.

Theorem 5.6. The global Markov ideal $I_{\mathrm{gl}\left(C_{N}\right)}$ is radical and has prime decomposition

$$
I_{\mathrm{gl}\left(C_{N}\right)}=I_{C_{N}} \cap \bigcap_{f \in \mathcal{B}_{4}} P_{f} .
$$

Proof. The intersection $J:=I_{C_{N}} \cap \bigcap_{f \in \mathcal{B}_{4}} P_{f}$ is a binomial ideal because, by Proposition 5.4, it is the radical of the binomial ideal $I_{\mathrm{gl}}\left(C_{N}\right)$ [13, Theorem 3.1]. Therefore, it suffices to consider an arbitrary binomial $p^{u}-p^{v} \in J$ and show that it is contained in $I_{\mathrm{gl}}\left(C_{N}\right)$. Since $J$ is homogeneous in the multigrading of the toric ideal $I_{C_{N}}$, there exists a sequence $v=u_{0}, u_{1}, \ldots, u_{r}=u$ such that $u_{i}-u_{i-1}$ is a move in the Markov basis $\mathcal{B}_{2} \cup \mathcal{B}_{4}$ of $I_{C_{N}}$. If only quadratic moves are necessary, then $p^{u}-p^{v} \in I$. Assume that $u_{i}-u_{i-1}$ is the first quartic move, and let $f$ be the corresponding quartic binomial. Then $p^{v}-p^{u_{i-1}} \in I_{\mathrm{gl}\left(C_{N}\right)} \subset P_{f}$, and hence $p^{u_{i-1}}-p^{u} \in P_{f}$. Therefore, $p^{u_{i-1}}$ must be divisible by a variable generating $P_{f}$ and, by definition, $p^{u_{i}}$ is divisible by the same variable. Hence, $p^{u_{i}}-p^{u_{i-1}} \in I_{\mathrm{gl}\left(C_{N}\right)}$ by Lemma 5.5. Iteration of this argument shows $p^{u}-p^{v} \in I_{\mathrm{gl}\left(C_{N}\right)}$.

Remark 5.7. The minimal primes of $I_{\mathrm{gl}\left(C_{N}\right)}$ are exactly witnessed by degree four binomials in the Markov basis of $I_{C_{N}}$. More precisely, if $f \in \mathcal{B}_{4}$, then $\left(I_{C_{N}}: f\right)$ is a minimal prime, and all minimal primes arise in this way.

Proof of Theorem 4.5. Let $P=P_{f}$ be one of the minimal primes, where $f$ is a quartic of the form (5.2). Then $m_{P}=f^{+} f^{-}$. Since $N \geq 4$, the quartic $f$ has at least two neighboring columns $i, i+1$ 
which are identical (up to symmetry). Hence, not all components of the $\{i, i+1\}$-marginal of the exponent vector of $m_{P}$ can be positive.

6. The complete bipartite graph $K_{2, N-2}$. In this section, we study the complete bipartite graph $K_{2, N-2}$ with vertex sets $\{1,2\}$, $\{3, \ldots, N\}$ and with $d_{1}=d_{2}=2$ and arbitrary $d_{3}, \ldots, d_{N}$. A Markov basis of the graphical model is presented in Theorem 6.1. Using this Markov basis, we compute a prime decomposition and show that $I_{\mathrm{gl}\left(K_{2, N-2}\right)}$ is radical (Theorem 6.5). With this decomposition, we prove that, for $N>4$, the complete bipartite graph does not satisfy the positive margins property (Theorem 6.6), but the interior point property (Theorem 6.8).

The set $\mathrm{gl}\left(K_{2, N-2}\right)$ consists of the CI statement $X_{1} \perp X_{2}$ | $\left\{X_{3}, \ldots, X_{N}\right\}$ and all statements $X_{A} \perp X_{B} \mid\left\{X_{1}, X_{2}, X_{C}\right\}$, where $A, B, C$ is a partition of $\{3, \ldots, N\}$. The variables of the polynomial ring $\mathbf{k}\left[p_{x}: x \in \mathcal{X}\right]$ can be arranged in a $\left(2 \times 2 \times d_{3} \times \cdots \times d_{n}\right)$-tensor $p=\left(p_{i j K}: i \in[2], j \in[2], K \in \prod_{s=3}^{N}\left[d_{s}\right]\right)$. Define $\left(d_{3} \times \cdots \times d_{N}\right)$ tensors $A^{i j}$ and $(2 \times 2)$-matrices $B^{K}$ via $A_{K}^{i j}=B_{i j}^{K}:=p_{i j K}$. Then $A^{i j}$ and $B^{K}$ are slices of $p$. The two sets of CI statements in $\operatorname{gl}\left(K_{2, N-2}\right)$ correspond to the two ideals

$$
I_{1}=\left\langle 2 \times 2 \text { minors of flattenings of } A^{i j}, i, j \in\{1,2\}\right\rangle
$$

and

$$
I_{2}=\left\langle 2 \times 2 \text { minors of } B^{K}, K \in \prod_{i=3}^{N}\left[d_{i}\right]\right\rangle .
$$

In $I_{1}$ we take all flattenings of the $(N-2)$-way tensor $A^{i j}$ down to a matrix and compute the $2 \times 2$ minors of those matrices. With this notation, we have $I_{\mathrm{gl}\left(K_{2, N-2}\right)}=I_{1}+I_{2}$. The quadratic generators of $I_{2}$ are of the form

$$
\left[\begin{array}{lll}
1 & 1 & K \\
2 & 2 & K
\end{array}\right]-\left[\begin{array}{lll}
1 & 2 & K \\
2 & 1 & K
\end{array}\right]
$$

and, up to symmetry, the generators of $I_{1}$ are of the form

$$
\left[\begin{array}{llll}
i & j & K & L \\
i & j & K^{\prime} & L^{\prime}
\end{array}\right]-\left[\begin{array}{cccc}
i & j & K^{\prime} & L \\
i & j & K & L^{\prime}
\end{array}\right]
$$


where $i, j, K, K^{\prime}, L, L^{\prime}$ are arbitrary in their respective domains (here, the symmetry says that we can permute the last $N-2$ columns).

Theorem 6.1. A Markov basis of the toric ideal $I_{K_{2, N-2}}$ with $d_{1}=d_{2}=2$ consists of the quadratic generators (6.1) of $I_{\mathrm{gl}\left(K_{2, N-2}\right)}$ and the quartic binomials

$$
B_{3 ; k_{1}, k_{2}}^{L_{11} L_{12} L_{21} L_{22}}:=\left[\begin{array}{cccc}
1 & 1 & k_{1} & L_{11} \\
1 & 2 & k_{2} & L_{12} \\
2 & 1 & k_{2} & L_{21} \\
2 & 2 & k_{1} & L_{22}
\end{array}\right]-\left[\begin{array}{cccc}
1 & 1 & k_{2} & L_{11} \\
1 & 2 & k_{1} & L_{12} \\
2 & 1 & k_{1} & L_{21} \\
2 & 2 & k_{2} & L_{22}
\end{array}\right]
$$

for all $k_{1}, k_{2} \in\left[d_{3}\right]$ and $L_{11}, L_{12}, L_{21}, L_{22} \in\left[d_{4}\right] \times \cdots \times\left[d_{n}\right]$, and the corresponding quartics $B_{a ; k_{1}, k_{2}}^{L_{11} L_{12} L_{21} L_{22}}$ for $a=4, \ldots, N$, where the roles of the columns 3 and a are exchanged in the above equation.

Proof. The proof is by induction on $N$. The base case $N=4$ is $[\mathbf{1 4}$, Corollary 2.2]. Suppose that Theorem 6.1 holds for some $N$. We show that it also holds for $N+1$. The graph $K_{2, N-1}$ is obtained by gluing the graph $K_{2, N-2}$ and the graph $K_{2,1}$ at the first two vertices. This is a codimension-one toric fiber product, which is slow-varying, since all quartic generators $B_{a ; k_{1}, k_{2}}^{L_{11} L_{12} L_{21} L_{22}}$ project to the zero polynomial when just considering their indices associated to the first two vertices, see $[\mathbf{1 4}$, subsection 5.3].

We first show that the set $\widetilde{\mathcal{B}}$ which consists of all quartics of the form $B_{a ; k_{1}, k_{2}}^{L_{11} L_{12} L_{21} L_{22}}$ and the quadratic moves of the form (6.1b) is a Markov basis of the associated codimension-zero toric fiber product, which is the graph model of the graph $\widetilde{K}_{2, N-1}$ with vertex set $[N+1]$ and edge set $\{(i, j): i<j \leq N+1, i \leq 2\}$. Again, this can be proved by induction: The induction base $\widetilde{K}_{2,1}=C_{3}$ is discussed in Remark 5.2. By [14, Theorem 5.4], a Markov basis of $I_{\tilde{K}_{2, N-1}}$ consists of the quadrics generating $I_{2}$ and lifts of elements of the Markov bases of $I_{\tilde{K}_{2, N-2}}$ and $I_{\tilde{K}_{2,1}}$. The lift of a quadratic generator of $I_{\tilde{K}_{2, N-1}}$ is a quadratic generator of $I_{2}$. The lift of a quartic generator of $I_{\tilde{K}_{2, N-1}}$ or $I_{\tilde{K}_{2,1}}$ is of the form $B_{a ; k_{1}, k_{2}}^{L_{11} L_{12} L_{21} L_{22}}$. This proves that $\widetilde{\mathcal{B}}$ is a Markov basis of $I_{\tilde{K}_{2, N-1}}$.

By [14, Theorem 5.10], we can obtain a Markov basis of $I_{K_{2, N-1}}$ from $\widetilde{\mathcal{B}}$ by adding additional quadrics of form $(6.1 \mathrm{~b})$ and moves obtained by 
gluing elements from the Markov bases of $I_{K_{2,1}}$ and $I_{K_{2, N-2}}$. Since $K_{2,1}$ is decomposable, the quadratic moves of form (6.1a) alone form a Markov basis of $I_{2,1}$ (no quartics are needed). These quadratic moves can only be glued with the corresponding quadratic generators from $I_{K_{2, N-1}}$, and this gluing procedure yields all quadratic moves of form (6.1a).

To sum up, the quartic moves and the quadratic moves of form (6.1b) belong to the associated codimension-one toric fiber product, and the quadratic moves of form (6.1a) arise iteratively from the quadratic generators of $I_{K_{2,1}}$.

Now we proceed to describe the other minimal primes of the ideal $I_{\mathrm{gl}\left(K_{2, N-2}\right)}$.

Lemma 6.2. Let $a, b \in\{3, \ldots, N\}$, and let $C \subset\left[d_{a}\right]$ and $D \subset\left[d_{b}\right]$. Then the ideal $P_{a, C, b, D}$ generated by $I_{\mathrm{gl}}\left(K_{2, N-2}\right)$ and the variables

$$
\begin{aligned}
\left\{p_{11 K}: K_{a} \in C\right\} & \cup\left\{p_{12 K}: K_{b} \in D\right\} \cup\left\{p_{21 K}: K_{b} \notin D\right\} \\
& \cup\left\{p_{22 K}: K_{a} \notin C\right\}
\end{aligned}
$$

is a prime ideal containing $I_{\mathrm{gl}}\left(K_{2, N-2}\right)$.

Proof. $P_{a, C, b, D}$ is prime since it is a sum of geometrically prime ideals which are defined in disjoint sets of variables. This can be seen as follows. First, the variables in $P_{a, C, b, D}$ generate a monomial prime ideal. Second, all binomial generators of $I_{2}$ are redundant modulo that ideal, i.e., they are implied by the variables in $P_{a, C, b, D}$. Third, let $f=p^{u}-p^{v}$ be a binomial generator of $I_{1}$. Then $p^{u}$ contains a variable generating $P_{a, C, b, D}$ if and only if $p^{v}$ contains a variable in $P_{a, C, b, D}$. The binomials in $I_{1}$ which are not implied by the variables in $P_{a, C, b, D}$ correspond to rank conditions on disjoint slices of the tensor $p$; hence, they generate a binomial prime ideal over any field.

Proposition 6.3. All minimal primes of $I_{\mathrm{gl}\left(K_{2, N-2}\right)}$ except the toric component $I_{K_{2, N-2}}$ are of the form $P_{a, C, b, D}$. Specifically:

1. If $N=4$, then the set of minimal primes consists of the toric component and all primes of the form $P_{a, C, a, D}$, where $a \in\{3,4\}$, $\varnothing \neq C \neq\left[d_{a}\right]$, and $\varnothing \neq D \neq\left[d_{a}\right]$. 
2. If $N>4$, then the set of minimal primes consists of the toric component and all primes of the form $P_{a, C, b, D}$, where $a, b \in\{3, \ldots, N\}$, $\varnothing \neq C \neq\left[d_{a}\right]$, and $\varnothing \neq D \neq\left[d_{b}\right]$.

The proof of Proposition 6.3 makes use of the following lemma.

Lemma 6.4. For any $K \in \prod_{i=3}^{n}\left[d_{i}\right]$,

$$
I_{\mathrm{gl}\left(K_{2, N-2}\right)}: p_{11 K} p_{22 K}=I_{\mathrm{gl}\left(K_{2, N-2}\right)}: p_{12 K} p_{21 K}=I_{K_{2, N-2}} .
$$

In particular, if $P$ is a minimal prime of $I_{\mathrm{gl}\left(K_{2, N-2}\right)}$ and not the toric component $I_{K_{2, N-2}}$, then $p_{11 K} p_{22 K} \in P$ and $p_{12 K} p_{21 K} \in P$.

Proof. We need to prove that both $p_{11 k L} p_{22 k L} B_{a ; i_{1} i_{2}}^{L_{11} L_{12} L_{21} L_{22}}$ and $p_{12 k L} p_{21 k L} B_{a ; i_{1} i_{2}}^{L_{11} L_{12} L_{21} L_{22}}$ belong to $I_{\mathrm{gl}\left(K_{2, N-2}\right)}$ and, by symmetry, it suffices to treat the first binomial. Moreover, by symmetry we may assume $a=3$. The calculation can be done using tableau notation:

$$
\begin{aligned}
& {\left[\begin{array}{cccc}
1 & 1 & k_{1} & L_{11} \\
1 & 2 & k_{2} & L_{12} \\
2 & 1 & k_{2} & L_{21} \\
2 & 2 & k_{1} & L_{22} \\
1 & 1 & k & L \\
2 & 2 & k & L
\end{array}\right]_{+}^{*} \rightarrow\left[\begin{array}{cccc}
1 & 1 & k & L_{11} \\
1 & 2 & k_{2} & L_{12} \\
2 & 1 & k_{2} & L_{21} \\
2 & 2 & k & L_{22} \\
1 & 1 & k_{1} & L \\
2 & 2 & k_{1} & L
\end{array}\right] *\left[\begin{array}{cccc}
1 & 1 & k & L_{11} \\
1 & 2 & k_{2} & L_{12} \\
2 & 1 & k_{2} & L_{21} \\
2 & 2 & k & L_{22} \\
1 & 2 & k_{1} & L \\
2 & 1 & k_{1} & L
\end{array}\right] *} \\
& \left.\rightarrow\left[\begin{array}{cccc}
1 & 1 & k & L_{11} \\
1 & 2 & k_{1} & L_{12} \\
2 & 1 & k_{1} & L_{21} \\
2 & 2 & k & L_{22} \\
1 & 2 & k_{2} & L \\
2 & 1 & k_{2} & L
\end{array}\right] *\left[\begin{array}{cccc}
1 & 1 & k & L_{11} \\
1 & 2 & k_{1} & L_{12} \\
2 & 1 & k_{1} & L_{21} \\
2 & 2 & k & L_{22} \\
1 & 1 & k_{2} & L \\
2 & 2 & k_{2} & L
\end{array}\right] \underset{*}{*} \rightarrow \begin{array}{cccc}
1 & 1 & k_{2} & L_{11} \\
1 & 2 & k_{1} & L_{12} \\
2 & 1 & k_{1} & L_{21} \\
& * \\
2 & 2 & k_{2} & L_{22} \\
1 & 1 & k & L \\
2 & 2 & k & L
\end{array}\right] .
\end{aligned}
$$

Here, the first tableau and the last tableau correspond to the two monomials of $p_{11 k L} p_{22 k L} B_{3 ; k_{1} k_{2}}^{L_{11} L_{12} L_{21} L_{22}}$.

Proof of Proposition 6.3. We use a set-theoretic argument. Let $p$ be any point in the variety of $I_{\mathrm{gl}\left(K_{2, N-2}\right)}$, and consider the $\left(d_{3} \times \cdots \times d_{N}\right)$ tensors $A^{i j}$ with $A_{K}^{i j}=p_{i j K}$. If no coordinate of $p$ vanishes, then $p$ is contained in the variety of $I_{K_{2, N-2}}$. Therefore, suppose $p_{i j K}=0$ 
for some $i j K \in \prod_{i=1}^{N}\left[d_{i}\right]$. The CI statements $\operatorname{gl}\left(K_{2, N-2}\right)$ imply that all $A^{i j}$ have rank one. Hence, there must be an index $a$ such that $p_{i j K^{\prime}}=0$ whenever $K^{\prime} \in \prod_{i=3}^{N}\left[d_{i}\right]$ satisfies $K_{a}^{\prime}=K_{a}$. In other words, for all $i, j \in\{1,2\}$, the pattern of zeros within $A^{i j}$ is a union of $(N-3)$ dimensional slices.

For each $a=3, \ldots, N$, let $E_{a}^{i j}$ be the largest subset of $\left[d_{a}\right]$ such that $p_{i j K}=0$ whenever $K_{a} \in E_{a}^{i j}$. Then $A_{K}^{i j} \neq 0$ if and only if $K \in\left(\left[d_{3}\right] \backslash E_{3}^{i j}\right) \times \cdots \times\left(\left[d_{N}\right] \backslash E_{N}^{i j}\right)$. By Lemma 6.4, if $p$ does not lie in the toric component, then $p_{\overline{i j} K}=A_{K}^{\overline{i j}}=0$ for all $K \in$ $\left(\left[d_{3}\right] \backslash E_{3}^{i j}\right) \times \cdots \times\left(\left[d_{N}\right] \backslash E_{N}^{i j}\right)$ (remember that $\overline{i j}$ denotes the "opposite" string to $i j$, obtained by exchanging $0 \leftrightarrow 1$ in each position). Again, each of these entries must be contained in an $(N-3)$-slice of zeros. Hence, there must be an index $a_{i j}$ such that $\left[d_{a_{i j}}\right] \backslash E_{a_{i j}}^{i j}$ is a subset of $E_{a_{i j}}^{\overline{i j}}$; for otherwise, if for each $a$ there exists $i_{a} \in\left(\left[d_{a}\right] \backslash E_{a}^{\overline{i j}}\right) \cap\left(\left[d_{a}\right] \backslash E_{a}^{i j}\right)$, then $p_{i j I} p_{\overline{i j} I} \neq 0$, where $I_{a}=i_{a}$ for all $a$. This implies that we can find subsets $C \subseteq\left[d_{a_{11}}\right], D \subseteq\left[d_{a_{12}}\right]$, such that $p \in V\left(P_{a_{11}, C, a_{12}, D}\right)$. This shows the first statement, and it remains to see that certain choices of $P_{a, C, b, D}$ do not appear.

If $C=\varnothing$, then $P_{a, C, b, D}$ contains the toric component: Indeed, $P_{a, \varnothing, b, D}$ contains all monomials of the form $p_{22 K}$, and hence $P_{a, \varnothing, b, D}$ contains all quartics. Therefore, $P_{a, \varnothing, b, D}$ is not a minimal prime, and the same is true if $C=\left[d_{a}\right], D=\varnothing$ or $D=\left[d_{b}\right]$. Similarly, if $N=4$, then $P_{3, C, 4, D}$ contains both monomials of any quartic.

It follows that all minimal primes are among the ideals $P_{a, C, b, D}$ listed in the statement of the theorem. It remains to show that all these ideals are indeed minimal primes. Note that each of these ideals contains a different set of variables of the same size, so they do not contain each other. Furthermore, they each leave out at least one of the quartic moves. Indeed, choose an index $c \in\{3, \ldots, N\} \backslash\{a, b\}$, choose $k_{1}, k_{2} \in\left[d_{c}\right]$, and choose $L_{11}, L_{12}, L_{21}, L_{22} \in \prod_{i>3, i \neq c}\left[d_{i}\right]$ such that $\left(L_{11}\right)_{a} \notin C,\left(L_{22}\right)_{a} \in C,\left(L_{12}\right)_{b} \notin D,\left(L_{21}\right)_{b} \in D$. Then $P_{a, C, b, D}$ does not contain $B_{a ; k_{1} k_{2}}^{L_{11} L_{12} L_{21} L_{22}}$, and so $I_{K_{2, N-2}} \nsubseteq P_{a, C, b, D}$.

Theorem 6.5. The global Markov ideal $I_{\mathrm{gl}\left(K_{2, N-2}\right)}$ is a radical ideal when $d_{1}=d_{2}=2$, with irredundant prime decomposition

$$
I_{\mathrm{gl}\left(K_{2, N-2}\right)}=I_{K_{2, N-2}} \cap \bigcap P_{a, C, b, D}
$$


with the intersection running over all $a, b \in\{3, \ldots, N\}, C \subset\left[d_{a}\right]$, $D \subset\left[d_{b}\right], C, D \neq \varnothing$. When $N=4$, we also require $a=b$.

Proof. Let $J$ be the intersection of the toric component with all minimal primes $P_{a, C, b, D}$. Lemma 6.2 shows $I \subseteq J$, and it remains to show the opposite inclusion. It suffices to consider binomials: By Proposition 6.3, the radical of $I$ equals $J$, and therefore $J$ is generated by binomials [13, Theorem 3.1].

Let $p^{u}-p^{v} \in J$. If there exists a prime $P_{a, C, b, D}$ such that $p^{u}$ does not contain any of the variables defining $P_{a, C, b, D}$, then $p^{u}-p^{v}$ actually belongs to the ideal generated by the binomial part of $P_{a, C, b, D}$, and hence $p^{u}-p^{v} \in I$. Therefore, we may assume in the following that, for any prime $P_{a, C, b, D}$, the monomial $p^{u}$ contains at least one of the variables defining $P_{a, C, b, D}$. Since $p^{u}-p^{v} \in I_{K_{2, N-2}}$, there is a decomposition $p^{u}-p^{v}=\sum_{i=1}^{r}\left(p^{u_{i-1}}-p^{u_{i}}\right)$, where $u_{0}=u, u_{r}=v$ and $u_{i-1}-u_{i}$ is an element of the Markov basis. If $u_{0}-u_{1}$ is a quadratic element of the Markov basis, then $p^{u}-p^{v}-p^{u}+p^{u_{1}}$ is an element of $J$ and belongs to $I$ if and only if $p^{u}-p^{v}$ belongs to $I$ (since $p^{u}-p^{u_{1}}$ is contained in $I$ as well as in each minimal prime).

Assume that $u_{0}-u_{1}$ corresponds to a quartic move, say $B_{3 ; k_{1} k_{2}}^{L_{11} L_{12} L_{21} L_{22}}$ for some $k_{1}, k_{2} \in[c]$ and $L_{11}, L_{12}, L_{21}, L_{22} \in[d]$. We use induction on the number of mismatches of $L_{11}$ and $L_{22}$ and the number of mismatches of $L_{12}$ and $L_{21}$ to show that we can replace this quartic with a combination of quadratic Markov moves. This shows that $p^{u}-p^{u_{1}}$ actually lies in $I$. If $L_{11}=L_{22}$, then the calculation

$$
\begin{aligned}
& {\left[\begin{array}{llll}
1 & 1 & k_{1} & L_{11} \\
1 & 2 & k_{2} & L_{12} \\
2 & 1 & k_{2} & L_{21} \\
2 & 2 & k_{1} & L_{11}
\end{array}\right]^{*} \longrightarrow\left[\begin{array}{cccc}
1 & 2 & k_{1} & L_{11} \\
1 & 2 & k_{2} & L_{12} \\
2 & 1 & k_{2} & L_{21} \\
2 & 1 & k_{1} & L_{11}
\end{array}\right] \begin{array}{c}
* \\
* \\
+
\end{array}} \\
& \longrightarrow\left[\begin{array}{llll}
1 & 2 & k_{2} & L_{11} \\
1 & 2 & k_{1} & L_{12} \\
2 & 1 & k_{1} & L_{21} \\
2 & 1 & k_{2} & L_{11}
\end{array}{ }^{*} \rightarrow\left[\begin{array}{cccc}
1 & 1 & k_{2} & L_{11} \\
1 & 2 & k_{1} & L_{12} \\
2 & 1 & k_{1} & L_{21} \\
2 & 2 & k_{2} & L_{11}
\end{array}\right]\right.
\end{aligned}
$$

shows that $B_{3 ; k_{1} k_{2} L_{11} L_{12} L_{11}}^{L_{1}}$ is a combination of quadratic Markov moves and hence lies in $I$. By symmetry, the same is true when $L_{12}=L_{21}$. Therefore, we may assume $L_{11} \neq L_{22}$ and $L_{12} \neq L_{21}$ in the following. 
As shown above, there exists a variable $p_{i j k L}$ that divides $p^{u}$ and $p^{u_{1}}$ (in particular, $p_{i j k L}$ is not involved in $B_{3 ; k_{1} k_{2}}^{L_{11} L_{12} L_{21} L_{22}}$ ). Without loss of generality, assume $i=j=2$. If there exists $a \geq 2$ such that $L_{a}=\left(L_{11}\right)_{a} \neq\left(L_{22}\right)_{a}$, then we can apply the moves

$$
\left[\begin{array}{cccc}
2 & 2 & k_{1} & L_{22} \\
2 & 2 & k & L
\end{array}\right]-\left[\begin{array}{cccc}
2 & 2 & k_{1} & L_{22}^{\prime} \\
2 & 2 & k & L^{\prime}
\end{array}\right]
$$

and

$$
\left[\begin{array}{cccc}
2 & 2 & k_{2} & L_{22} \\
2 & 2 & k & L
\end{array}\right]-\left[\begin{array}{cccc}
2 & 2 & k_{2} & L_{22}^{\prime} \\
2 & 2 & k & L^{\prime}
\end{array}\right]
$$

with

$$
\left(L_{22}^{\prime}\right)_{b}= \begin{cases}\left(L_{22}\right)_{b} & \text { if } b \neq a \\ L_{a} & \text { if } b=a\end{cases}
$$

and

$$
L_{b}^{\prime}= \begin{cases}L_{b} & \text { if } b \neq a, \\ \left(L_{22}\right)_{a} & \text { if } b=a,\end{cases}
$$

to $p^{u}$ and $p^{u_{1}}$. This effectively replaces $L_{22}$ by $L_{22}^{\prime}$, and $L_{11}$ and $L_{22}^{\prime}$ agree in more components than $L_{11}$ and $L_{22}$.

By symmetry, if there exist $i, j \in\{0,1\}$ and $a \geq 2$ with $\left.L=L_{i j}\right)_{a} \neq$ $\left(L_{\overline{i j}}\right)_{a}$, then we can apply quadratic moves to make $L_{i j}$ and $L_{\overline{i j}}$ more similar to each other. Now we may assume that each variable $p_{i j k L}$ that divides $p^{u}$ satisfies $L_{a} \neq\left(L_{\overline{i j}}\right)_{a}$. We show that it is still possible for some $i, j$ to reduce the number of mismatches between $L_{i j}$ and $L_{\overline{i j}}$.

Choose indices $a$ and $b$ such that $\left(L_{11}\right)_{a} \neq\left(L_{22}\right)_{a}$ and $\left(L_{12}\right)_{b} \neq$ $\left(L_{21}\right)_{b}$. We claim that, in this case, there exist $k_{3}, k_{4} \in\left[d_{3}\right]$ and $L_{5}, L_{6} \in \prod_{i=4}^{n}\left[d_{i}\right]$ such that

- either $\left(L_{5}\right)_{a}=\left(L_{6}\right)_{a}$ and $p_{11 k_{3} L_{5}} p_{22 k_{4} L_{6}}$ divides $p^{u}$,

- or $\left(L_{5}\right)_{b}=\left(L_{6}\right)_{b}$ and $p_{12 k_{3} L_{5}} p_{21 k_{4} L_{6}}$ divides $p^{u}$.

Otherwise, $p^{u}$ would contain no defining variable of the prime $P_{a, C, b, D}$ with

$$
\begin{aligned}
& C=\left\{l \in\left[d_{a}\right]: p_{11 k L} \text { does not divide } p^{u} \text { for all } k \in\left[d_{1}\right],\right. \\
& \left.\qquad L \in \prod_{s=2}^{n}\left[d_{s}\right] \text { with } L_{a}=l\right\},
\end{aligned}
$$




$$
\begin{aligned}
& D=\left\{l \in\left[d_{b}\right]: p_{12 k L} \text { does not divide } p^{u} \text { for all } k \in\left[d_{1}\right],\right. \\
& \left.\qquad L \in \prod_{s=2}^{n}\left[d_{s}\right] \text { with } L_{b}=l\right\}
\end{aligned}
$$

(note that if $N=4$, then $a=b$ ). By symmetry, it suffices to consider the first case, i.e., $\left(L_{5}\right)_{a}=\left(L_{6}\right)_{a}$ and $p_{11 k_{3} L_{5}} p_{22 k_{4} L_{6}}$ divides $p^{u}$. We can then apply the moves

$$
\left[\begin{array}{cccc}
1 & 1 & k_{1} & L_{11} \\
1 & 1 & k_{3} & L_{5}
\end{array}\right]-\left[\begin{array}{cccc}
1 & 1 & k_{1} & L_{11}^{\prime} \\
1 & 1 & k_{3} & L_{5}^{\prime}
\end{array}\right]
$$

and

$$
\left[\begin{array}{cccc}
2 & 2 & k_{1} & L_{22} \\
2 & 2 & k_{3} & L_{6}
\end{array}\right]-\left[\begin{array}{cccc}
2 & 2 & k_{1} & L_{22}^{\prime} \\
2 & 2 & k_{3} & L_{6}^{\prime}
\end{array}\right]
$$

with

$$
\left(L_{11}^{\prime}\right)_{c}=\left\{\begin{array}{ll}
\left(L_{11}\right)_{c} & \text { if } c \neq a, \\
\left(L_{5}\right)_{a} & \text { if } c=a,
\end{array} \quad\left(L_{5}\right)_{c}^{\prime}= \begin{cases}\left(L_{5}\right)_{c} & \text { if } c \neq a \\
\left(L_{22}\right)_{a} & \text { if } c=a\end{cases}\right.
$$

and

$$
\left(L_{22}^{\prime}\right)_{c}=\left\{\begin{array}{ll}
\left(L_{22}\right)_{c} & \text { if } c \neq a, \\
\left(L_{6}\right)_{a} & \text { if } c=a,
\end{array}\left(L_{6}\right)_{c}^{\prime}= \begin{cases}\left(L_{6}\right)_{c} & \text { if } c \neq a \\
\left(L_{22}\right)_{a} & \text { if } c=a,\end{cases}\right.
$$

to $p^{u}$ and $p^{u_{1}}$. This effectively replaces $L_{11}$ by $L_{11}^{\prime}$ and $L_{22}$ by $L_{22}^{\prime}$, and $L_{11}^{\prime}$ and $L_{22}^{\prime}$ agree in more components than $L_{11}$ and $L_{22}$. This proves the induction step and shows that $p^{u}-p^{v}$ lies in $I$.

With this primary decomposition, we can analyze the positive margins property.

Theorem 6.6. For $N \geq 4$, the complete bipartite graph $K_{2, N-2}$, where the first group of nodes is binary, has the positive margins property if and only if $N=4$.

Proof. We check the condition in Lemma 4.1. If $N=4$, then each minimal prime is of the form $P=P_{a, C, a, D}$. Because of the symmetry we may assume $a=3$. Then

$$
m_{P}=\prod_{k, l: k \notin C} p_{11 k l} \prod_{k, l: k \notin D} p_{12 k l} \prod_{k, l: k \in D} p_{21 k l} \prod_{k, l: k \in C} p_{22 k l} .
$$


Suppose that $C$ and $D$ intersect. Then the $\{1,3\}$-marginal of the exponent vector of $m_{P}$ is not strictly positive, since any variable $p_{1 j k l}$ that divides $m_{P}$ satisfies $k \notin C \cap D$. Similarly, if $C$ and $D$ do not intersect, then $C$ intersects the complement of $D$, and hence the $\{2,3\}$ marginal cannot be strictly positive.

If $N>4$, then consider a prime of the form $P=P_{3, C, 4, D}$. Then

$$
m_{P}=\prod_{K: K_{a} \notin C} p_{11 K} \prod_{K: K_{b} \notin D} p_{12 K} \prod_{K: K_{b} \in D} p_{21 K} \prod_{K: K_{a} \in C} p_{22 K},
$$

and the exponent vector has strictly positive margins. Indeed, take for example the $\{1, a\}$-marginal. For any $k \in\left[d_{a}\right]$, choose $K, L \in$ $\left[d_{3}\right] \times \cdots \times\left[d_{N}\right]$ such that $K_{b} \notin D, L_{b} \in D$ and $K_{a}=k=L_{a}$. Then $p_{12 K} p_{21 L}$ divides $m_{P}$, and hence the $\{1, k\}$-count and the $\{2, k\}$-count of the $\{1, a\}$-marginal are larger than zero.

Finally, we want to prove that $K_{2, N-2}$ satisfies the interior point property. We first describe additional inequalities of the marginal cone.

Lemma 6.7. Let $N \geq 4$, and assume $d_{1}=d_{2}=2$. For any table $u \in \mathbf{N}^{n}$, denote by $y=A u$ the vector of $K_{2, N-2}$-marginals, which has components $y_{k l}^{i j}$ for $i=1,2, j=3, \ldots, N$, and $(k, l) \in[2] \times\left[d_{j}\right]$. Let $a, b$ be such that $3 \leq a<b \leq N$, and let $C \subset\left[d_{a}\right], D \subset\left[d_{b}\right]$ be non-empty subsets such that $C \neq\left[d_{a}\right]$ and $D \neq\left[d_{b}\right]$. For any choice of $a, b, C, D$,

$$
\sum_{k \in C} y_{1 k}^{1 a}+\sum_{k \notin C} y_{2 k}^{2 a}+\sum_{l \in D} y_{1 l}^{2 b}-\sum_{l \in D} y_{1 l}^{1 b} \geq 0
$$

Proof. It suffices to show that each unit vector in $\mathbf{N}^{n}$ satisfies (6.2). Consider the unit vector $e_{x}$ corresponding to $x \in \mathcal{X}$. If the last summand $\sum_{l \in D} y_{1 l}^{1 b}\left(e_{x}\right)$ vanishes, then (6.2) holds. Otherwise, $x_{1}=1$ and $x_{l} \in D$, and so this sum equals one. In this case, at least one of the following three possibilities happens. Either $x_{a} \in C$, or $x_{a} \notin C$ and $x_{2}=2$, or $x_{2}=1$. In any case, $\sum_{k \in C} y_{1 k}^{1 a}+\sum_{k \notin C} y_{2 k}^{2 a}+\sum_{l \in D} y_{1 l}^{2 b} \geq 1$, and so (6.2) holds.

Theorem 6.8. Assume that $d_{1}=d_{2}=2$. If $u \in \mathbf{N}^{n}$ has strictly positive $K_{2, N-2}$-margins, and if $u$ satisfies all inequalities of the form 
(6.2) with strict inequality, then the fiber of $u$ is connected by quadratic moves.

Proof. We apply Lemma 2.5. Let $P=P_{a, C, b, D}$ be a minimal prime. If $a=b$, then the proof of Theorem 6.6 shows that the exponent vector $u_{P}$ of $m_{P}$ has at least one vanishing marginal. If $a \neq b$, then a direct verification shows that $u_{P}$ satisfies

$$
\sum_{k \in C} y_{1 k}^{1 a}+\sum_{k \notin C} y_{2 k}^{2 a}+\sum_{l \in D} y_{1 l}^{2 b}-\sum_{l \in D} y_{1 l}^{1 b}=0 \text {. }
$$

Proof of Theorem 4.6. Combine Theorems 6.6 and 6.8 .

Acknowledgments. In November 2011 the first and second authors worked intensively on this project during a two-week "Research in Pairs" stay at Mathematisches Forschungsinstitut Oberwolfach.

\section{REFERENCES}

1. 4ti2-A software package for algebraic, geometric and combinatorial problems on linear spaces, available at www.4ti2.de, 2007.

2. J. Besag, Spatial interaction and the statistical analysis of lattice systems, J. Roy. Stat. Soc. 36 (1974), 192-236.

3. René Birkner, Polyhedra: A package for computations with convex polyhedral objects, J. Software Alg. Geom. 1 (2009), 11-15.

4. Nicolas Bourbaki, Éléments de mathématique, in Algèbre, Hermann, 1950.

5. Florentina Bunea and Julian Besag, MCMC in $i \times j \times k$ contingency tables, in Monte Carlo methods, Neal Madras, ed., Fields Inst. Comm. 26, AMS and Fields Institute, 2000.

6. Yuguo Chen, Ian H. Dinwoodie and Seth Sullivant, Sequential importance sampling for multiway tables, Ann. Stat. 34 (2006), 523-545.

7. Yuguo Chen, Ian Dinwoodie and Ruriko Yoshida, Markov chains, quotient ideals, and connectivity with positive margins, in Algebraic and geometric methods in statistics, Pablo Gibilisco, Eva Riccomagno, Maria Piera Rogantin and Henry P. Wynn, eds., Cambridge University Press, Cambridge, 2010.

8. József Dénes and A.D. Keedwell, Latin squares and their applications, Academic Press, New York, 1974.

9. Mike Develin and Seth Sullivant, Markov bases of binary graph models, Ann. Comb. 7 (2003), 441-466.

10. Persi Diaconis, David Eisenbud and Bernd Sturmfels, Lattice walks and primary decomposition, in Mathematical essays in honor of Gian-Carlo Rota, B. Sagan and R. Stanley, eds., Progr. Math. 161, Birkhauser, Boston, 1998. 
11. Persi Diaconis and Bernd Sturmfels, Algebraic algorithms for sampling from conditional distributions, Ann. Stat. 26 (1998), 363-397.

12. Mathias Drton, Bernd Sturmfels and Seth Sullivant, Lectures on algebraic statistics, Oberwolfach Sem. 39, Birkhäuser, Springer, Berlin, 2009.

13. David Eisenbud and Bernd Sturmfels, Binomial ideals, Duke Math. J. 84 (1996), 1-45.

14. Alexander Engström, Thomas Kahle and Seth Sullivant, Multigraded commutative algebra of graph decompositions, J. Alg. Comb. 39 (2014), 335-372.

15. Dan Geiger, Christopher Meek and Bernd Sturmfels, On the toric algebra of graphical models, Ann. Stat. 34 (2006), 1463-1492.

16. Daniel R. Grayson and Michael E. Stillman, Macaulay2, A software system for research in algebraic geometry, Available at http://www.math.uiuc.edu/Macaulay2/.

17. Jürgen Herzog, Takayuki Hibi, Freyja Hreinsdóttir, Thomas Kahle and Johannes Rauh, Binomial edge ideals and conditional independence statements, Adv. Appl. Math. 45 (2010), 317-333.

18. Thomas Kahle, Decompositions of binomial ideals, J. Software Alg. Geom. 4 (2012), 1-5.

19. - GraphBinomials, A library for walks on graphs on monomials, available from https://github.com/tom111/GraphBinomials, 2012.

20. Thomas Kahle and Ezra Miller, Decompositions of commutative monoid congruences and binomial ideals, 2011, arXiv:1107.4699.

21. Steffen L. Lauritzen, Graphical models, Oxford Stat. Sci. Ser., Oxford University Press, Oxford, 1996.

22. Jesús A. De Loera and Shmuel Onn, Markov bases of three-way tables are arbitrarily complicated, J. Symb. Comp. 41 (2006), 173-181.

23. Peter N. Malkin, Truncated Markov bases and Gröbner bases for integer programming, manuscript, 2006.

24. Johannes Rauh, Generalized binomial edge ideals, Adv. Appl. Math. 50 (2013), 409-414.

25. Johannes Rauh and Nihat Ay, Robustness canalyzing functions and systems design, Theor. Biosci. 133 (2014), 63-78.

26. Johannes Rauh and Thomas Kahle, The Markov bases database, http://markovbases.de.

27. Ronald C. Read and Robin J. Wilson, An atlas of graphs, Clarendon Press, New York, 1998.

28. Bernd Sturmfels, Gröbner bases of toric varieties, Tōhoku Math. J. 43 (1991), 249-261.

29. - Gröbner bases and convex polytopes, University Lecture Series 8, American Mathematical Society, Providence, RI, 1996.

30. - Solving systems of polynomial equations, CBMS 97, American Mathematical Society, Providence, 2002.

31. Seth Sullivant, Toric fiber products, J. Algebra 316 (2007), 560-577.

32. Irena Swanson and Amelia Taylor, Minimal primes of ideals arising from conditional independence statements, J. Alg. 392 (2013), 299-314. 
OvGU Magdeburg, FMA-IAG, Universitätsplatz 2, 39106 Magdeburg, GERMANY

Email address: thomas.kahle@ovgu.de

Max Planck Institute for Mathematics in the Sciences, Inselstrasse 22, D-04103 Leipzig, Germany

Email address: jrauh@mis.mpg.de

Department of Mathematics, North Carolina State University, Box 8205, Raleigh, NC 27695

Email address: smsulli2@ncsu.edu 